\title{
Relativistic Quantum Statistical Mechanics in Two-Dimensional Space-Time
}

\author{
Raphael Høegh-Krohn \\ Institute of Mathematics, University of Oslo, Blindern, Oslo, Norway
}

Received November 11, 1973; in revised form May 6, 1974

\begin{abstract}
We construct for a boson field in two-dimensional space-time with polynomial or exponential interactions and without cut-offs, the positive temperature state or the Gibbs state at temperature $1 / \beta$. We prove that at positive temperatures i.e. $\beta<\infty$, there is no phase transitions and the thermodynamic limit exists and is unique for all interactions. It turns out that the Schwinger functions for the Gibbs state at temperature $1 / \beta$ is after interchange of space and time equal to the Schwinger functions for the vacuum or temperature zero state for the field in a periodic box of length $\beta$, and the lowest eigenvalue for the energy of the field in a periodic box is simply related to the pressure in the Gibbs state at temperature $1 / \beta$.
\end{abstract}

\section{Introduction}

Although the study of the statistical mechanics for quantum systems has made good progress the last ten years [1], the progress has been best for discrete systems of lattice systems. The main difficulty in connection with continuous systems has been that the group of time automorphisms $\alpha_{t}$ for the Schrödinger particles is non local. The consequence of this non locallity is that the infinite system of interacting Schrödinger particles do not agree well with the generally accepted picture of a quantum statistical mechanics described in terms of a local $C^{*}$-algebra or a $C^{*}$-algebra of local operators, on which the time acts as a group $\alpha_{t}$ of $C^{*}$-automorphisms. Hence we get a somewhat discouraging situation, that the only known realistic model of a statistical quantum mechanics, namely the system of interacting Schrödinger particles, does not conform to the highly developed abstract theory of quantum statistical mechanics.

For this very reason the question of studying relativistic particles instead of Schrödinger particles appears quite natural, since in any relativistic theory there should be an upper bound for the propagation speed and this would force the group of time automorphisms $\alpha_{t}$ to be local. This is the motivation for this paper.

We know free relativisic particles are described by free quantum fields and it is a general belief that interacting relativistic particles are 
to be described by the interacting quantum fields. Unfortunately at present time the interacting quantum fields are well understood only in the case of scalar fields in two space time dimensions with polynomial or exponential interactions.

In the case of weak polynomial interaction in two space-time dimensions we have by the recent results on the particle structure by Glimm, Jaffe, and Spencer [2], that in fact the interacting quantum field is a description of a system of infinitely many interacting relativistic scalar particles. Hence we see that at least in this case the interacting relativistic particles are described by the interacting quantum fields, so that the statistical mechanics of interacting relativistic particles is nothing but the statistical mechanics of the interacting quantum fields. So that for instance the vacuum state for the interacting quantum field is nothing but the zero temperature state for the infinite system of interacting relativistic particles described by the field. In the case of weak polynomial interaction and strong exponential interactions in two space time dimensions one also has a very clear picture of what happens with the vacuum in the infinite volume limit, or as we would like to say it here, one has a very clear picture of the thermodynamic limit in the case of temperature zero. For the weak polynomial interactions this was treated by Glimm, Jaffe, and Spencer [2], and in the case of exponential interactions by Albeverio and Høegh-Krohn [3]. Hence good candidates for a quantum statistical mechanics of interacting relativistic particles are the polynomial and exponential interactions in two spacetime dimensions.

In this paper I study the thermodynamic limit of the positive temperature Gibbs state for the polynomial and exponential interactions in two space time dimensions.

The method I use is strongly influenced by recent works by Nelson [4], and may be denoted as the Markoff field approach. The Markoff field approach was also a main ingredient in [3] and played also a certain role in [2]. One of the advantages of the Markoff field approach is to make available for quantum fields the methods of classical statistical mechanics, and this is the way it is used in [3], leaning heavily on the work of Guerra, Rosen, and Simon [5]; that introduces a framework which describes the Markoff fields as Ising ferromegnetic systems.

The way the Markoff field approach is used here is somewhat different. In this paper we use the Markoff field to transform the problem about the thermodynamic limit for the Gibbs state at temperature $1 / \beta$ for the relativistic quantum statistical system into the problem of the uniqueness of the vacuum for the system in a periodic box of length $\beta$.

In fact it turns out that for any of the interactions we consider, namely the polynomial and the exponential interaction, the Markoff 
fields for the Gibbs state at temperature $1 / \beta$ is the Markoff field on the cylinder $S_{\beta} \times R$, where $S_{\beta}$ is a circle of length $\beta$, that corresponds to the Markoff field for the vacuum in the plane $R \times R$, and this last Markoff field is the limit of the first one as the temperature $1 / \beta$ goes to zero.

Using this method it is proved that the thermodynamic limit for the Gibbs state exists for all positive temperatures $1 / \beta$ and all interactions considered i.e. for strong exponential interactions as well as strong polynomial interactions in two space time dimensions.

We see that this is in strong contrast to the vacuum or temperature zero case for the polynomial interactions, where Glimm and Spencer were only able to prove the existence of the infinite volume limit for weak interactions, and from Dobrushin and Minlos [6] we know by now that this is best possible, in fact for any even polynomial interaction in two space-time dimensions they get that the thermodynamic limit is not unique in the temperature zero case for strong enough interactions. The reason for this difference is the above mentioned fact that while for the temperature zero case we have a Markoff field in the plane $R \times R$ so that the problem is two dimensional, we have for positive temperature a Markoff field on the cylinder $S_{\beta} \times R$ so that the problem is essentially one dimensional, and therefore in a sense much simpler.

The Gibbs state at positive temperature $1 / \beta$ is of course not invariant under the Lorentz group since it is given in terms of the energy operator. There is however, a Lorentz invariant analogy of the Gibbs state at positive temperature $1 / \beta$. But this Lorentz invariant Gibbs state is only to be found in a closed universe, the so called De Sitter universe, and it will lead too far to give the construction of the positive temperature state in the De Sitter universe in this paper. This will be dealt with separately in a forthcoming paper.

\section{The Gibbs-State for the Harmonic Oscillator}

Consider the self adjoint operator

$$
H_{0}=-\frac{1}{2} \Delta+\frac{1}{2}\left(x, A^{2} x\right)-\frac{1}{2} \operatorname{tr} A
$$

on the Hilbert space $\mathscr{H}=L_{2}\left(R^{N}\right)$, where $\Delta=\sum_{i=1}^{N} \frac{\partial^{2}}{\partial x_{i}^{2}}$ and $A$ is a real symmetric $N \times N$ matrix bounded below by a positive constant, $A \geqq c I$, $c>0, x \in R^{N}$ and (,) is the natural inner product in $R^{N}$.

Let $\lambda_{1}, \ldots, \lambda_{N}$ be the eigenvalues of $A$. It is well known that $H_{0}$ has discrete spectrum consisting of the points of the form

$$
\sum_{k=1}^{n} \lambda_{i_{k}}
$$


and zero. Hence for a positive $\beta, e^{-\beta H_{0}}$ is of trace class and we get

hence

$$
\operatorname{tr} e^{-\beta H_{0}}=\sum_{n_{1} \geqq 0, \ldots, n_{N} \geqq 0} \mathrm{e}^{-\beta \sum_{i=1}^{N} n_{\imath} \lambda_{i}},
$$

$$
\operatorname{tr} e^{-\beta H_{0}}=\prod_{i=1}^{N}\left(1-e^{-\beta \lambda_{i}}\right)^{-1}
$$

Let $V(x) \geqq-b$ be a real measurable function bounded below such that

$$
H=H_{0}+V(x)
$$

is essentially self adjoint. We say that $H$ is the Hamiltonian for the anharmonic oscillator. From $V \geqq-b$ we get $H \geqq H_{0}-b$, which gives us that $H$ has discrete spectrum and together with (2.2) it gives a lower bound for the eigenvalues of $H$, which is transformed into an upper bound for the eigenvalues of $e^{-\beta H}$. Hence $e^{-\beta H}$ is of trace class. Therefore we may form the normal state $\omega_{\beta}$ on the von Neumann algebra $B(\mathscr{H})$ of all bounded operators on $\mathscr{H}$, given by

$$
\omega_{\beta}(A)=\left(\operatorname{tr} e^{-\beta H}\right)^{-1} \operatorname{tr}\left(A e^{-\beta H}\right)
$$

for $A \in B(\mathscr{H}) . \omega_{\beta}$ is called the Gibbs-state for the anharmonic oscillator.

By the Feynman-Kac formula we know that the kernel $e^{-\beta H}(x, y)$ of the operator $e^{-\beta H}$ is given by

$$
e^{-\beta H}(x, y)=E_{(x, y)}^{\beta}\left[e^{-\int_{0}^{\beta} U(x(\tau)) d \tau}\right],
$$

with $U(x)=\frac{1}{2}\left(x, A^{2} x\right)-\frac{1}{2} \operatorname{tr} A+V(x)$ and $E_{(x, y)}^{\beta}$ is the conditional expectation with respect to the Brownian motion in $R_{N}$ given that $x(0)=x$ and $x(\beta)=y$. So that $E_{(0,0)}^{\beta}$ is the expectation with respect to the normal distribution indexed by the real Hilbert space $h$ of continuous functions $x(\tau)$ from $[0, \beta]$ into $R^{N}$, such that $x(0)=x(\beta)=0$ and the norm square

$$
\int_{0}^{\beta}\left(\frac{d x(\tau)}{d \tau}, \frac{d x(\tau)}{d \tau}\right) d \tau
$$

is finite.

Consider the Hilbert space $L_{2}\left([0, \beta] ; R^{N}\right)$ of $L_{2}$-integrable functions from $[0, \beta]$ into $R^{N}$, and let $k_{i, j}(s, t)$ be the kernel of the inverse operator of the self adjoint operator $-\frac{d^{2}}{d \tau^{2}}$ with boundary conditions $x(0)=x(\beta)=0$ on $L_{2}\left([0, \beta] ; R^{N}\right)$. Then

$$
k_{i j}(s, t)=k(s, t) \delta_{i j}
$$


and

$$
k(s, t)=\left\{\begin{array}{ll}
\frac{1}{\beta} s(\beta-t) & s \leqq t \\
\frac{1}{\beta}(\beta-s) t & s \geqq t
\end{array} .\right.
$$

The normal distribution indexed by $h$ is the same as the Gaussian process with mean zero and covariance function $k_{i j}(s, t)$.

It is well known that the Brownian motion, hence also the process above has support on the continuous functions from $[0, \beta]$ into $R^{N}$.

In terms of the measures introduced above $E_{(x, y)}^{\beta}$ is the expectation with respect to the measure obtained from the normal distribution indexed by $h$ by a transformation on the continuous functions from $[0, \beta]$ into $R^{N}$ given by

$$
x(\tau) \rightarrow x+\frac{\tau}{\beta}(y-x)+x(\tau) .
$$

From (2.6) we now get that the kernel $e^{-\beta H}(x, y)$ is a continuous function of $x$ and $y$. It is well known in that case that $\operatorname{tr} e^{-\beta H}=\int e^{-\beta H}(x, x) d x$, which together with (2.6) gives

$$
\operatorname{tr} e^{-\beta H}=\int E_{(x, x)}^{\beta}\left[e^{-\int_{0}^{\beta} U(x(\tau)) d \tau}\right] d x .
$$

By (2.9) $E_{(x, x)}^{\beta}$ is the expectation with respect to the measure on the continuous periodic functions from $[0, \beta]$ into $R^{N}$ obtained from the normal distribution indexed by $h$ by the transformation $x(\tau) \rightarrow x(\tau)+x$.

Since $U(x)=\frac{1}{2}\left(x, A^{2} x\right)-\frac{1}{2} \operatorname{tr} A+V(x)$ we have that

$$
\int_{R^{N}} E_{(x, x)}^{\beta}\left[e^{-\int_{0}^{\beta} U(x(\tau)) d \tau}\right] d x=\int_{R^{N}} E_{(x, x)}^{\beta}\left[e^{-\frac{1}{2} \int_{0}^{\beta}\left(x(\tau), A^{2} x(\tau)\right)} \cdot e^{-\int^{\beta} V(x(\tau)) d \tau}\right] d x .
$$

On the other hand we easily verify that for any real continuous function $F$ defined on the space of continuous periodic functions from $[0, \beta]$ into $R^{N}$

$$
\int_{R^{N}} E_{(x, x)}^{\beta}\left[e^{-\frac{1}{2} \int\left(x(\tau), A^{2} x(\tau)\right) d \tau} F\right] d x=C E^{\beta}[F]
$$

where $E^{\beta}$ is the expectation with respect to the normal distribution indexed by the real Hilbert space $g$ of continuous periodic functions from $[0, \beta]$ into $R^{N}, x(0)=x(\beta)$, such that the norm square

$$
\int_{0}^{\beta}\left[\left(\frac{d x(\tau)}{d \tau}, \frac{d x(\tau)}{d \tau}\right)+\left(x(\tau), A^{2} x(\tau)\right)\right] d \tau
$$


is finite. $C$ is some positive constant independent of $F$. By setting $V=0$ in (2.11) we get that $C=\operatorname{tr} e^{-\beta H_{0}}$.

We have thus proved the following formula

$$
\operatorname{tr} e^{-\beta H}=\operatorname{tr} e^{-\beta H_{0}} E^{\beta}\left[e^{-\int_{0}^{\beta} V(x(\tau)) d \tau}\right]
$$

where $E^{\beta}$ is the expectation with respect to the normal distribution indexed by the real Hilbertspace $g$ of periodic functions from $[0, \beta]$ into $R^{N}$ with norm square given by (2.13). Now $\operatorname{tr} e^{-\beta H_{0}}$ is given by (2.3), and since $1-e^{-\beta \lambda_{i}}$ are the eigenvalues of the real symmetric matrix $1-e^{-\beta A}$ we get from (2.3) that

$$
\operatorname{tr} e^{-\beta H_{0}}=\left|1-e^{-\beta A}\right|^{-1}
$$

where $\left|1-e^{-\beta A}\right|$ is the determinant of the matrix $1-e^{-\beta A}$. Hence (2.14) may be written

$$
\operatorname{tr} e^{-\beta H}=\left|1-e^{-\beta A}\right|^{-1} E^{\beta}\left[e^{-\int_{0}^{\beta} V(x(\tau)) d \tau}\right] .
$$

Let now $F_{i} \in B(\mathscr{H}) i=0, \ldots, n$ be multiplication operators by bounded continuous functions $F_{i}(x), i=0, \ldots, n$, and let $0=s_{0} \leqq s_{1} \cdots \leqq s_{n-1} \leqq s_{n}=\beta$.

Consider the operator

$$
F_{0} e^{-s_{1} H} F_{1} e^{-\left(s_{2}-s_{1}\right) H} \ldots F_{n} e^{-\left(s_{n}-s_{n-1}\right) H} .
$$

From (2.6) we have that the kernel of $F_{i} e^{-\left(s_{i+1}-s_{1}\right) H}$ is given by

$$
F_{i}(x) e^{-\left(s_{i+1}-s_{i}\right) H}(x, y)=E_{(x, y)}^{s_{2+1}-s_{i}}\left[F_{i}(x(0)) e^{-\int_{0}^{s_{i+1}-s_{i}} U(x(\tau)) d \tau}\right] .
$$

Since the Brownian motion is a homogeneous process, (2.18) may be written in the form

$$
E_{(x, y)}^{\left[s_{i}, s_{i+1}\right]}\left[F_{i}\left(x\left(s_{i}\right)\right) e^{-\int_{s}^{s_{i+1}} U(x(\tau)) d \tau}\right]
$$

where $E_{(x, y)}^{\left[s_{i}, s_{i}+1\right]}$ is the conditional expectation with respect to the Brownian motion given that $x\left(s_{i}\right)=x$ and $x\left(s_{i+1}\right)=y$. Utilizing now the Markovian properties of the Browian motion we get that the kernel of the operator $F_{0} e^{-s_{1} H} F_{1} e^{-\left(s_{2}-s_{1}\right) H}$ is given by

$$
\begin{array}{r}
\int d z E_{(x, z)}^{\left[0, s_{1}\right]}\left[F_{0}(x(0)) e^{-\int_{0}^{s_{2}} U(x(\tau)) d \tau}\right] \cdot E_{(z, y)}^{\left[s_{1}, s_{2}\right]}\left[F_{1}\left(x\left(s_{1}\right)\right) e^{-\int_{s_{1}}^{s_{1}} U(x(\tau)) d \tau}\right] \\
=E_{(x, y)}^{\left[0, s_{2}\right]}\left[F_{0}(x(0)) F_{1}\left(x\left(s_{1}\right)\right) e^{-\int_{0}^{s_{2}} U(x(\tau)) d \tau}\right] .
\end{array}
$$


By induction we get the kernel of the operator (2.17) is given by

$$
E_{(x, y)}^{\beta}\left[\prod_{i=0}^{n-1} F_{i}\left(x\left(s_{i}\right)\right) e^{-\int^{\beta} U(x(\tau)) d \tau}\right] .
$$

By computing the trace of this kernel in the same way as we computed the trace (2.16) of the kernel (2.6), we prove the following theorem.

Theorem 2.1. Let $F_{i} \in B(\mathscr{H}) i=0, \ldots, n$ be multiplication operators by bounded continuous functions $F_{i}(x), i=0, \ldots, n$, let $0=s_{0} \leqq s_{1} \cdots \leqq s_{n}=\beta$, and let $H$ be the Hamiltonian for the anharmonic oscillator (2.4) then

$$
\begin{aligned}
& \operatorname{tr}\left(F_{0} e^{-s_{1} H} F_{1} e^{-\left(s_{2}-s_{1}\right) H} \ldots F_{n-1} e^{-\left(\beta-s_{n-1}\right) H}\right) \\
& =\left|1-e^{-\beta A}\right|^{-1} E^{\beta}\left[e^{-\int^{\beta} V(x(\tau)) d \tau} \prod_{i=0}^{n-1} F_{i}\left(x\left(s_{i}\right)\right)\right]
\end{aligned}
$$

where $\left|1-e^{-\beta A}\right|$ is the determinant of the matrix $1-e^{-\beta A}$ and $E^{\beta}$ is the expectation with respect to the normal distribution indexed by the real Hilbert space $g$ of continuous periodic functions from $[0, \beta]$ into $R_{N}$, $x(0)=x(\beta)$, with norm square equal to

$$
\int_{0}^{\beta}\left[\left(\frac{d x(\tau)}{d \tau}, \frac{d x(\tau)}{d \tau}\right)+\left(x(\tau), A^{2} x(\tau)\right)\right] d \tau .
$$

By a direct calculation one easily verifies the following remark.

Remark. The expectation $E^{\beta}$ in the theorem above is the expectation with respect to the homogeneous Gaussian process on a circle of length $\beta$ with values in $R^{N}$ given by the covariance matrix $E^{\beta}\left(x_{i}(s) x_{j}(t)\right)$ equal to the matrix

$$
\frac{1}{\beta} A^{-2}+\frac{2}{\beta} \sum_{n=1}^{\infty}\left(\frac{4 \pi^{2}}{\beta^{2}} n^{2}+A^{2}\right)^{-1} \cos \frac{2 \pi}{\beta} n(s-t) .
$$

Summing up this series we get a more explicit expression for the covariance matrix

$$
E^{\beta}\left(x_{i}(0) x_{j}(t)\right)=\left(2 A\left(e^{\beta A}-1\right)\right)^{-1}\left[e^{(\beta-t) A}+e^{t A}\right]
$$

for $0 \leqq t \leqq \beta$.

Let $\alpha_{t}$ be the $C^{*}$-automorphism of $B(\mathscr{H})$ defined by

then

$$
\begin{aligned}
& \alpha_{t}(B)=e^{-i t H} B e^{i t H} \\
& \operatorname{tr}\left(B \alpha_{t}(C) e^{-\beta H}\right) \\
= & \operatorname{tr}\left(B e^{-i t H} C e^{-(\beta-i t) H}\right) \\
= & \operatorname{tr}\left(C e^{-(\beta-i t) H} B e^{-i t H}\right)
\end{aligned}
$$

is analytic in $t$ in the strip $-\beta<\operatorname{Imt}<0$, with boundary values at real $t$ equal to $\operatorname{tr}\left(B \alpha_{t}(C) e^{-\beta H}\right)$ and at $t-i \beta$ equal to $\operatorname{tr}\left(C \alpha_{-t}(B) e^{-\beta H}\right)$. 
Consider now an operator of the form (2.17).

$$
\operatorname{tr}\left(F_{0} e^{-s_{1} H} F_{1} e^{-\left(s_{2}-s_{1}\right) H} \ldots F_{n-1} e^{-(\beta-n-1) H}\right)
$$

is obviously analytic in the domain $0<\operatorname{Res}_{1}<\operatorname{Res}_{2} \cdots<\operatorname{Res}_{n-1}<\beta$ with boundary values at $\operatorname{Res}_{i}=0, i=0, \ldots, n$ which are continuous and uniformly bounded and given by

$$
\operatorname{tr}\left(F_{0} \alpha_{t_{1}}\left(F_{1}\right) \alpha_{t_{2}}\left(F_{2}\right) \ldots \alpha_{t_{n-1}}\left(F_{n-1}\right) e^{-\beta H}\right)
$$

for $s_{k}=i t_{k}, k=1, \ldots, n-1$.

The continuity of (2.28) follows from the strong continuity of $e^{i t H}$.

Lemma 2.1. Let $t_{i} \in R$ and $F_{i}$ be bounded continuous functions on $R^{N}$, then $B(\mathscr{H})$ is the smallest strongly closed linear space of operators that contains all operators of the form $\alpha_{t_{1}}\left(F_{1}\right) \cdot \alpha_{t_{2}}\left(F_{2}\right) \ldots \alpha_{t_{n}}\left(F_{n}\right)$.

Proof. Since the smallest strongly closed linear space containing the operators above is obviously a strongly closed $C^{*}$-algebra of operators, it is enough to prove that if $B \in B(\mathscr{H})$ commute with $\alpha_{t}(F)$ for all $t$ and all continuous functions $F$ then $B=\lambda I$. Therefore assume $\left[B, \alpha_{t}(F)\right]=0$ for all $t$ and $F$. Then $\left[\alpha_{t}(B), F\right]=0$ for all $F$ and $t$, hence $\alpha_{t}(B)$ is a multiplication operator by an $L_{\infty}$-function for all $t$. Hence for any real $L_{\infty}$-function $W$

so that

$$
e^{i s W} \alpha_{t}(B) e^{-i s W}=\alpha_{t}(B)
$$

By the Trotter-Kato product formula

$$
\text { strong } \lim _{n \rightarrow \infty}\left(e^{i \frac{t}{n} H} e^{-i \frac{t}{n} W}\right)^{n}=e^{i t(H-W)}
$$

and therefore by $(2.30)$

$$
\alpha_{t}(B)=e^{-i t(H-W)} B e^{i t(H-W)} .
$$

By letting $W(x)$ increase to $U(x)$, we get that $(1+H-W)^{-1}$ increase to $\left(1-\frac{1}{2} \Delta\right)^{-1}$ so that $(1+H-W)^{-1}$ converge strongly to $\left(1-\frac{1}{2} \Delta\right)^{-1}$ and so by the semigroup theorem $e^{i t(H-W)}$ converge strongly to $e^{-i \frac{t}{2} \Delta}$. Hence by (2.32) we get that

$$
\alpha_{t}(B)=e^{i \frac{t}{2} \Delta} B e^{-i \frac{t}{2} \Delta} .
$$

Since $\alpha_{t}(B)$ is a multiplication operator for all $t$ we have that $B$ is a multiplication operator. But it is easy to see that if $B$ is not equal to $\lambda I$, then $e^{i \frac{t}{2} \Delta} B e^{-i \frac{t}{2} \Delta}$ is not a multiplication operator. This proves the lemma.

Using that $\omega_{\beta}$ is a normal state we get the following theorem

Theorem 2.2. Let $B$ and $C$ be in $B(\mathscr{H})$, then

$$
\omega_{\beta}\left(B \alpha_{t}(C)\right)=\omega_{\beta}\left(\alpha_{-t}(B) \cdot C\right)
$$


is analytic in the strip $-\beta<\operatorname{Im} t<0$, and continuous and uniformly bounded in $-\beta \leqq \operatorname{Im} t \leqq 0$. The boundary values satisfy the KMS condition

for real $t$.

$$
\omega_{\beta}\left(B \alpha_{t-i \beta}(C)\right)=\omega_{\beta}\left(C \alpha_{-t}(B)\right)
$$

Moreover, any operator $B$ in $B(\mathscr{H})$ may be approximated strongly by linear combinations of operators of the form $\alpha_{t_{1}}\left(F_{1}\right) \alpha_{t_{2}}\left(F_{2}\right) \ldots \alpha_{t_{n}}\left(F_{n}\right)$, where $F_{1}, \ldots, F_{n}$ are multiplication operators by continuous functions $F_{1}(x), \ldots, F_{n}(x)$, hence $\omega_{\beta}(B)$ will also be approximated by the same linear combinations of $\omega_{\beta}\left(\alpha_{t_{1}}\left(F_{1}\right) \alpha_{t_{2}}\left(F_{2}\right) \ldots \alpha_{t_{n}}\left(F_{n}\right)\right)$. Furthermore $\omega_{\beta}\left(F_{0} \alpha_{t_{1}}\left(F_{1}\right)\right.$ $\left.\ldots \alpha_{t_{n}}\left(F_{n}\right)\right)$ is analytic in $0>\operatorname{Im} t_{1}>\cdots>\operatorname{Im} t_{n}>-\beta$ and its value for $t_{k}=-i s_{k} k=1, \ldots, n$ with $0=s_{0} \leqq s_{1} \leqq \cdots \leqq s_{n} \leqq \beta$ is given by

$$
\begin{aligned}
\omega_{\beta}\left(F_{0} \alpha_{-i s_{1}}\left(F_{1}\right) \ldots \alpha_{-i s_{n}}\left(F_{n}\right)\right) \\
=\left(E^{\beta}\left[e^{-\int_{0}^{\beta} V(x(\tau)) d \tau}\right]\right)^{-1} E^{\beta}\left[\prod_{i=0}^{n} F_{i}\left(x\left(s_{i}\right)\right) e^{-\int^{\beta} V(x(\tau)) d \tau}\right]
\end{aligned}
$$

where $E^{\beta}$ is the expectation given in Theorem 2.1.

\section{The Gibbs-State for the Free Scalar Quantum Field}

Let $\Lambda \subset R^{n}$ be a bounded domain in $R^{n}$ with a regular boundary $\partial \Lambda$. Let $A_{\Lambda}^{2}=-\Delta+m^{2}$ where $\Delta$ is the Laplace operator in $\Lambda$ with some self adjoint boundary conditions on $\partial \Lambda$. If the constant functions satisfy the boundary we shall assume that $m>0$ if not only that $m \geqq 0$, so that in any case, $A_{A}^{2}$ is a self adjoint operator on the real Hilbert space $L_{2}^{R}(\Lambda)$ and

$$
A_{\Lambda} \geqq c I, \quad c>0 .
$$

It is well known that $A_{A}$ has discrete spectrum and that $e^{-\beta A_{A}}$ is of trace class for all $\beta>0$, so that the Fredholm determinant $\left|1-e^{-\beta A_{A}}\right|$ exists, and by (3.1) it is different from zero.

Let $h_{A}$ be the real Hilbert space $D\left(A_{\Lambda}\right) \subset L_{2}^{R}(\Lambda)$ with norm square equal to

$$
2\left(x, A_{\Lambda} x\right)
$$

for $x \in D\left(A_{A}\right)$ where (,) is the inner product in $L_{2}^{R}(\Lambda)$. $h_{A}$ depends of course also on the boundary conditions on $\partial \Lambda$. Let now $L_{2}\left(h_{1}\right)$ be the complex Hilbert space of $L_{2}$ integrable functions with respect to the normal distribution indexed by the real Hilbert space $h_{\Lambda}$.

The Hamiltonian $H_{0}(\Lambda)$ for the free scalar field in $\Lambda$ with mass $m$ and the given boundary conditions is a self adjoint operator on $L_{2}\left(h_{A}\right)$ 
which is denoted by

$$
H_{0}(\Lambda)=-\frac{1}{2} \Delta_{\Lambda}+\frac{1}{2}\left(x, A_{\Lambda}^{2} x\right)=\frac{1}{2} \operatorname{tr} A_{\Lambda}
$$

where $\Delta_{\Lambda}$ denotes the Laplace operator on $L_{2}^{R}(\Lambda)$ and $($,$) is the inner$ product on $L_{2}^{R}(\Lambda)$. (3.3) is not a definition of $H_{0}(\Lambda)$ but just a convenient notation. We shall now give the proper definition of $H_{0}(\Lambda)$.

Let $\left\{e_{k}\right\}_{k=1}^{\infty}$ be the complete orthonormal base in $L_{2}^{R}(\Lambda)$ of eigenfunctions for $A_{\Lambda}$

$$
A_{\Lambda} e_{k}=\lambda_{k} e_{k} .
$$

The probability space for the normal distribution $d n_{h_{A}}$ indexed by the real Hilbert space $h_{A}$ is then in a natural way identified with infinite product of the probability spaces for the one dimensional normal distributions

so that

$$
d n_{\lambda_{k}}=\left(\frac{\lambda_{k}}{\pi}\right)^{\frac{1}{2}} e^{-\lambda_{k} x_{k}^{2}} d x_{k},
$$

$$
d n_{h_{\lambda}}=\chi_{k=1}^{\infty} d n_{\lambda_{k}}
$$

Hence $L_{2}\left(h_{\Lambda}\right)$ may be identified with the infinite tensor product

$$
L_{2}\left(h_{A}\right)=\bigotimes_{k=1}^{\infty} L_{2}\left(d n_{\lambda_{k}}\right)
$$

relative to the vectors $f_{k} \in L_{2}\left(d n_{\lambda_{k}}\right)$ given by $f_{k}\left(x_{k}\right)=1$. Now $L_{2}\left(d n_{\lambda_{k}}\right)$ may be identified with $L_{2}(R)$ by the identification

$$
g\left(x_{k}\right) \leftrightarrow\left(\frac{\lambda_{k}}{\pi}\right)^{\frac{1}{4}} e^{-\frac{1}{2} \lambda_{k} x_{k}^{2}} g\left(x_{k}\right)
$$

for $g \in L_{2}\left(d n_{\lambda_{k}}\right)$. Therefore $L_{2}\left(h_{A}\right)$ may be identified with the infinite tensor product

$$
L_{2}\left(h_{\Lambda}\right)=\bigotimes_{k=1}^{\infty} L_{2}(R)
$$

relative to the vectors $g_{k} \in L_{2}(R)$ given by

$$
g_{k}\left(x_{k}\right)=\left(\frac{\lambda_{k}}{\pi}\right)^{\frac{1}{4}} e^{-\frac{1}{2} \lambda_{k} x_{k}^{2}} .
$$

Let now $H_{\lambda_{1}}$ be the Hamiltonian for a one dimensional harmonic oscillator given by

$$
H_{\lambda_{k}}=-\frac{1}{2} \frac{d^{2}}{d x_{k}^{2}}+\frac{\lambda_{k}^{2}}{2} x_{k}^{2}-\frac{\lambda_{k}}{2}
$$


as a self adjoint operator on the $k$-th component in the tensor product (3.9). $e^{i t H_{k}}$ is then a strongly continuous unitary group on the $k$-th component wich leaves the vector $g_{k}$ invariant. It is then well known that the infinite tensor product $\bigotimes_{k=1}^{\infty} e^{i t H_{k}}$ exists and forms a strongly continuous unitary group on the infinite tensor product (3.9). We now define $H_{0}(\Lambda)$ as the self adjoint infinitesimal generator of this unitary group on $L_{2}\left(h_{\Lambda}\right)$.

Definition.

$$
e^{i t H_{0}(\Lambda)}=\bigotimes_{k=1}^{\infty} e^{i t H_{k}}
$$

relative to the tensor decomposition (3.9).

From this definition we get immediately that $e^{-\beta H_{0}(\Lambda)}$ is of trace class for $\beta>0$ and that

$$
\operatorname{tr} e^{-\beta H_{0}(\Lambda)}=\left|1-e^{-\beta A_{\Lambda}}\right|^{-1} .
$$

We now define the Gibbs-state for the free scalar field of mass $m$ in $\Lambda$ with the given boundary conditions by

$$
\omega_{\beta}^{0}(\Lambda)(B)=\left(\operatorname{tr} e^{-\beta H_{0}(\Lambda)}\right)^{-1} \operatorname{tr}\left(B e^{-\beta H_{0}(\Lambda)}\right)
$$

for any $B$ in the $C^{*}$-algebra $B\left(L_{2}\left(h_{\Lambda}\right)\right)$.

Let $F$ be a bounded continuous function on $R^{N}$. From (3.9) we get the following tensor decomposition

$$
L_{2}\left(h_{A}\right)=L_{2}\left(R^{N}\right) \otimes\left[\bigotimes_{k=N+1}^{\infty} L_{2}(R)\right]
$$

where the infinite tensor product here is also relative to the vectors (3.10). $F$ may then be identified with an element $F \otimes 1$ of $B\left(L_{2}\left(h_{A}\right)\right)$ in accordance with the tensor decomposition (3.15). We shall denote this element in $B\left(L_{2}\left(h_{A}\right)\right)$ also by $F$. By $L_{\infty}\left(h_{A}\right)$ we shall understand the maximal abelian algebra in $B\left(L_{2}(h)\right)$ containing all bounded continuous functions $F$ on $R^{N}$ for all values of $N$. It is obvious that $L_{\infty}\left(h_{A}\right)$ is the space of $L_{\infty}$-functions on the probability space associated with the normal distribution indexed by $h_{\Lambda}$.

Let $H_{0}^{N}(\Lambda)$ be the infinitesimal generator of the unitary group on $L_{2}\left(R^{N}\right)$ given by

$$
e^{i t H_{0}^{N}(\Lambda)}=\bigotimes_{k=1}^{N} e^{i t H_{k}},
$$

and let $F_{0}, \ldots, F_{n-1}$ be bounded continuous functions on $R^{N}$ and $0=s_{0} \leqq \cdots \leqq s_{n}=\beta$. It follows then immediately from the definition 
(3.12) of $H_{0}(\Lambda)$ that if we consider $F_{0}, \ldots, F_{n-1}$ as elements in $L_{\infty}\left(h_{\Lambda}\right)$ then

$$
\begin{aligned}
& \operatorname{tr}\left(F_{0} e^{-s_{1} H_{0}(\Lambda)} F_{1} e^{-\left(s_{2}-s_{1}\right) H_{0}(\Lambda)} \ldots F_{n-1} e^{-\left(\beta-s_{n}-1\right) H_{0}(\Lambda)}\right) \\
& \quad=\prod_{k=N+1}^{\infty}\left(1-e^{-\beta \lambda_{k}}\right)^{-1} \operatorname{tr}_{N}\left(F_{0} e^{-s_{1} H_{0}^{N}(\Lambda)} \ldots F_{n-1} e^{-\left(\beta-s_{n-1}\right) H_{0}^{N}(\Lambda)}\right)
\end{aligned}
$$

where $\operatorname{tr}_{N}$ is the trace in $L_{2}\left(R^{N}\right)$. By Theorem 2.1

$$
\begin{aligned}
\operatorname{tr}_{N}\left(F_{0} e^{-s_{1} H_{0}^{N}(\Lambda)} \ldots F_{n-1} e^{-\left(\beta-s_{n-1}\right) H_{0}^{N}(\Lambda)}\right) & \\
& =\prod_{k=1}^{N}\left(1-e^{-\beta \lambda_{k}}\right)^{-1} E_{N}^{\beta}\left[\prod_{i=0}^{n-1} F\left(x\left(s_{i}\right)\right)\right]
\end{aligned}
$$

where $E_{N}^{\beta}$ is the expectation with respect to the normal distribution indexed by the real Hilbert space $g_{N}$ of continuous functions from the circle $S_{\beta}$ of length $\beta$ into $R^{N}$ with norm square equal to

$$
\sum_{k=1}^{N} \int_{0}^{\beta}\left[\left(\frac{d x_{k}}{d \tau}\right)^{2}+\lambda_{k}^{2}\left(x_{k}(\tau)\right)^{2}\right] d \tau
$$

Let $g_{\beta}(\Lambda)$ be the real Hilbert space of functions from $S_{\beta} \times \Lambda$ into $R$ such that the norm square

$$
\int_{0}^{\beta} \int_{\Lambda}\left[\left(\frac{\partial \varepsilon}{\partial t}\right)^{2}+\sum_{i=1}^{n}\left(\frac{\partial \xi}{\partial x_{i}}\right)^{2}+m^{2} \xi^{2}\right] d x d t
$$

is finite and such that for all $t, 0 \leqq t \leqq \beta, \xi(x, t)$ satisfies the self adjoint boundary conditions given by $A^{2}$. If we consider $\xi(x, t)$ as a function $\eta(t)$ from $S_{\beta}$ into $L_{2}^{R}(\Lambda)$, then (3.20) takes the form

$$
\int_{0}^{\beta}\left[\left(\frac{d \eta}{d \tau}, \frac{d \eta}{d \tau}\right)+\left(\eta(\tau), A_{\Lambda}^{2} \eta(\tau)\right)\right] d \tau \text {. }
$$

From (3.19) it then follows that $g_{N}$ is a closed subspace of $g_{\beta}(\Lambda)$ generated by all functions $\eta(t)$ such that $\eta(t)$ is in the subspace of $L_{2}^{R}(\Lambda)$ generated by the $N$ first eigenvectors $e_{1}, \ldots, e_{N}$ of $A$, for all $t, 0 \leqq t \leqq \beta$. This together with (3.17) and (3.18) gives then that

$$
\begin{aligned}
\operatorname{tr}\left(F_{0} e^{-s_{1} H_{0}(\Lambda)} F_{1} e^{-\left(s_{2}-s_{1}\right) H_{0}(\Lambda)} \ldots F_{n-1} e^{-\left(\beta-s_{n-1}\right) H_{0}(\Lambda)}\right) \\
=\mid 1-e^{-\left.\beta A_{\Lambda}\right|^{-1}} E_{\Lambda}^{\beta}\left[\prod_{i=0}^{n-1} F_{i}\left(\eta\left(s_{i}\right)\right)\right],
\end{aligned}
$$

where $E_{A}^{\beta}$ is the expectation with respect to the normal distribution indexed by the real Hilbert space $g_{\beta}(\Lambda)$.

Since the bounded continuous functions on $R^{N}$ are obviously weakly dense in $L_{\infty}\left(h_{A}\right)$, we may extend (3.22) to arbitrary $F_{0}, \ldots, F_{n-1}$ in $L_{\infty}\left(h_{A}\right)$. Utilizing the remark following Theorem 2.1 we may also compute the covariance for $E_{\Lambda}^{\beta}$. We have thus the following theorem. 

then

Theorem 3.1. Let $F_{0}, \ldots, F_{m-1}$ be in $L_{\infty}\left(h_{A}\right)$, and $0=s_{0} \leqq s_{1} \leqq \cdots \leqq s_{m}=\beta$,

$$
\begin{aligned}
& \operatorname{tr}\left(F_{0} e^{-s_{1} H_{0}(\Lambda)} F_{1} e^{-\left(s_{2}-s_{1}\right) H_{0}(\Lambda)} \ldots F_{m-1} e^{-\left(\beta-s_{m-1}\right) H_{0}(\Lambda)}\right) \\
& =\left|1-e^{-\beta A_{\Lambda}}\right|^{-1} E_{\Lambda}^{\beta}\left[\prod_{i=0}^{m-1} F_{i}\left(\eta\left(s_{i}\right)\right)\right]
\end{aligned}
$$

where $E^{\beta}$ is the expectation with respect to the normal distribution indexed by the real Hilbert space $g_{\beta}(\Lambda)$, of functions from the circle $S_{\beta}$ of length $\beta$ into $D\left(A_{A}\right)$ with norm square equal to

$$
\int_{0}^{\beta}\left[\left(\frac{d \eta}{d \tau}, \frac{d \eta}{d \tau}\right)+\left(\eta(\tau), A_{\Lambda}^{2} \eta(\tau)\right)\right] d \tau,
$$

where (, ) is the inner product in $L_{2}^{R}(\Lambda) . E^{\beta}$ may also be characterized as the Gaussian distribution with mean zero and co-variance which is invariant on $S_{\beta}$ and given by

$$
E_{\Lambda}^{\beta}[(\varphi, \eta(0))(\psi, \eta(t))]=\left(\varphi,\left(2 A\left(1-e^{-\beta A_{\Lambda}}\right)^{-1}\left(e^{-t A_{\Lambda}}+e^{-(\beta-t) A_{A}}\right) \psi\right)\right)
$$

for $0 \leqq t \leqq \beta$, where $\varphi$ and $\psi$ are in $L_{2}(\Lambda)$ and $($,$) is the inner product in L_{2}(\Lambda)$.

If we consider the elements in $g_{\beta}(\Lambda)$ as functions $\xi(x, t)$ from $S_{\beta} \times \Lambda$ into $R$ satisfying the proper boundary conditions on $\partial \Lambda$, then $E_{\Lambda}^{\beta}$ may be characterized as the expectation with respect to the generalized Gaussian prosess on $S_{\beta} \times \Lambda$ with covariance function given by

$$
E_{\Lambda}^{\beta}(\xi(x, s) \xi(y, t))=G_{\Lambda}^{\beta}(x, y, s-t)
$$

where $G_{A}^{\beta}(x, y, s-t)$ is the Greensfunction for the self adjoint operator $-\frac{\partial^{2}}{\partial t^{2}}-\Delta+m^{2}$ on $S_{\beta} \times \Lambda$ with the corresponding self adjoint boundary conditions on $\partial \Lambda$.

We define now the $C^{*}$-automorphism $\alpha_{t}^{0}(\Lambda)$ on $B\left(L_{2}\left(h_{\Lambda}\right)\right)$ by

$$
\alpha_{t}^{0}(\Lambda)(B)=e^{-i t H_{0}(\Lambda)} B e^{i t H_{0}(\Lambda)} .
$$

Then for $B$ and $C$ in $B\left(L_{2}\left(h_{A}\right)\right)$ we have that

$$
\omega_{\beta}^{0}(\Lambda)\left(B \cdot \alpha_{t}^{0}(\Lambda)(C)\right)=\omega_{\beta}^{0}(\Lambda)\left(\alpha_{-t}^{0}(\Lambda)(B) \cdot C\right) .
$$

Moreover (3.24) is an analytic function of $t$ in the strip $-\beta<\operatorname{Im} t<0$, which is continuous and uniformly bounded in $-\beta \leqq \operatorname{Im} t \leqq 0$, and the boundary values satisfies the $K M S$ condition

$$
\omega_{\beta}^{0}(\Lambda)\left(B \cdot \alpha_{t-i \beta}^{0}(\Lambda)(C)\right)=\omega_{\beta}^{0}(\Lambda)\left(C \alpha_{-t}^{0}(\Lambda)(B)\right) .
$$

Further more, if $F_{0}, \ldots, F_{n}$ are in $L_{\infty}\left(h_{\Lambda}\right)$ then

$$
\omega_{\beta}^{0}(\Lambda)\left(F_{0} \alpha_{t_{1}}^{0}(\Lambda)\left(F_{1}\right) \ldots \alpha_{t_{m}}^{0}(\Lambda)\left(F_{m}\right)\right)
$$


is analytic in $0>\operatorname{Im} t_{1}>\cdots>\operatorname{Im} t_{m}>-\beta$ and continuous and uniformly bounded in $0 \geqq \operatorname{Im} t_{1} \geqq \cdots \geqq \operatorname{Im} t_{m} \geqq-\beta$, and its value for $t_{k}=-i s_{k}, k=1, \ldots, m$ with $0=s_{0} \leqq s_{1} \ldots \leqq s_{m}=\beta$ is given by

$$
\omega_{\beta}^{0}(\Lambda)\left(F_{0} \alpha_{-i s_{1}}^{0}(\Lambda)\left(F_{1}\right) \ldots \alpha_{-i s_{m}}^{0}(\Lambda)\left(F_{m}\right)\right)=E_{\Lambda}^{\beta}\left[\prod_{k=0}^{m} F_{k}\left(\eta\left(s_{k}\right)\right)\right] .
$$

Let $\mathcal{O} C R^{n}$ be a bounded open set in $R^{n}$ and let $\varphi \in C_{0}^{\infty}(\mathcal{O})$ and real. It is easily seen that the normal distribution indexed by $h_{A}$ is quasiinvariant under the transformation $\eta \rightarrow \eta+\varphi$, if $\mathscr{O} \subset \Lambda$. Hence this transformation induces a unitary transformation $U(\varphi)$ on $L_{2}\left(h_{A}\right)$. Let $V(\varphi)$ be the unitary transformation of multiplication by the $L_{\infty}\left(h_{A}\right)$ function $e^{i(\varphi, \eta)}$ where $($,$) is the inner product in L_{2}(\Lambda) . \mathscr{A}_{0}(\mathcal{O})$ is then the smallest norm closed algebra in $B\left(L_{2}\left(h_{A}\right)\right)$ containing $U(\varphi)$ and $V(\varphi)$ for all real $\varphi \in C_{0}^{\infty}(\mathcal{O})$. Since $\mathscr{A}_{0}(\mathcal{O})$ is a $C^{*}$-algebra which is faithfully represented in each $B\left(L_{2}\left(h_{A}\right)\right)$ for all $\Lambda \supset \mathcal{O}, \mathscr{A}_{0}(\mathcal{O})$ will not depend on the particular $\Lambda$ as soon as $\Lambda \supset \mathcal{O}$.

By (3.2) the normal distribution indexed by $h_{A}$, may be characterized as the generalized Gaussian process with mean zero and covariance function $G_{A}(x, y)$, where $G_{A}(x, y)$ is the Greens function for the selfadjoint operator $A_{1}$. Let now $\mathcal{O}$ be contained in the interior of $\Lambda_{1}$ and $\Lambda_{2}$. Since $G_{A_{1}}(x, y)-G_{A_{2}}(x, y)$ is a smooth function for $x$ and $y$ in $\mathcal{O}$, it follows that the conditional expectations of the normal distributions indexed by $h_{A_{1}}$ and $h_{A_{2}}$ with respect to the $\sigma$-algebra generated by functions of the form $(\varphi, \eta)$ with $\varphi \in C_{0}^{\infty}(\mathcal{O})$ are equivalent measures. From this it immediately follows that $\mathscr{A}_{0}(\mathcal{O})$ has equivalent representations in $B\left(L_{2}\left(h_{A_{1}}\right)\right)$ and $B\left(L_{2}\left(h_{A_{2}}\right)\right)$, so that the strong closure $\overline{\mathscr{A}}(\mathcal{O})$ of $\mathscr{A}_{0}(\mathcal{O})$ in $B\left(L_{2}\left(h_{A}\right)\right)$ is independent of $A$ as soon as $\mathcal{O}$ is contained in the interior of $\Lambda$. We have obviously that $\overline{\mathscr{A}}\left(\mathcal{O}_{1}\right) \subseteq \overline{\mathscr{A}}\left(\mathcal{O}_{2}\right)$ if $\mathcal{O}_{1} \subset \mathcal{O}_{2}$. Let $\overline{\mathscr{A}}$ be the norm closure of $\cup\left\{\mathscr{A}(\mathcal{O}) \mid \mathcal{O} \subset R^{n}\right\}$.

Let now $B \in \overline{\mathscr{A}}(\mathcal{O})$. It is then well known that $\alpha_{t}^{0}(\Lambda)(B) \in \overline{\mathscr{A}}\left(\mathcal{O}_{t}\right)$, where $\mathcal{O}_{t}$ is the open set of points with distance smaller than $t$ from $\mathcal{O}$, and that $\alpha_{t}^{0}(\Lambda)(B)$ is independent of $\Lambda$ as soon as $\mathcal{O}_{t}$ is contained in the interior of $\Lambda$. We shall denote this independent value by $\alpha_{t}^{0}(B) . \alpha_{t}^{0}$ is then a $C^{*}$-isomorphism from $\overline{\mathscr{A}}(\mathcal{O})$ into $\overline{\mathscr{A}}\left(\mathcal{O}_{t}\right)$ for any $\mathcal{O}$, hence it extends to a $C^{*}$-automorphism of $\overline{\mathscr{A}}$.

Now let $\mathscr{F}(\mathcal{O})$ be all functions in $L_{\infty}\left(h_{A}\right)$ of the form $f\left(\left(\eta, \varphi_{1}\right),\left(\left(\eta, \varphi_{2}\right)\right.\right.$, $\left.\ldots,\left(\eta, \varphi_{N}\right)\right)$, where $f\left(x_{1}, \ldots, x_{N}\right)$ is bounded continuous function on $R^{N}$ and $\varphi_{1}, \ldots, \varphi_{N}$ is in $C_{0}^{\infty}(\mathcal{O})$. We denote by $\mathscr{A}_{0}$ the smallest norm closed $C^{*}$-algebra in $\overline{\mathscr{A}}$ which contains all operators of the form $\alpha_{t}^{0}(F)$, for $F \in \mathscr{F}(\mathcal{O})$ for some $\mathcal{O} . \overline{\mathscr{A}}_{0}$ is then obviously invariant under $\alpha_{t}^{0}$, and we shall say that $\mathscr{A}_{0}$ is the local algebra for the free field. 
Let $F_{0}, \ldots, F_{m}$ be in $\mathscr{F}(\mathcal{O})$ for some $\mathcal{O}$. We shall then show that

$$
\omega_{\beta}^{0}(\Lambda)\left(F_{0} \alpha_{t_{1}}^{0}\left(F_{1}\right) \ldots \alpha_{t_{m}}^{0}\left(F_{m}\right)\right)
$$

converge as $\Lambda$ tends to $R^{n}$ in such a way that it finally contains all bounded sets, independently of the boundary conditions on $\partial \Lambda$. To see this, choose any $T>0$. Then for $\left|t_{k}\right| \leqq T, k=1, \ldots, m$, since $\Lambda$ finally contains any bounded set, we have that from a certain point on $\mathcal{O}_{T}$ is contained in the interior of $\Lambda$, but then (3.27) is equal to

$$
\omega_{\beta}^{0}(\Lambda)\left(F_{0} \alpha_{t_{1}}^{0}(\Lambda)\left(F_{1}\right) \ldots \alpha_{t_{m}}^{0}\left(F_{m}\right)\right) .
$$

Now (3.28) is analytic in $0>\operatorname{Im} t_{1}>\cdots>\operatorname{Im} t_{m}>-\beta$ and uniformly bounded and continuous in $0 \geqq \operatorname{Im} t_{1} \geqq \cdots \geqq \operatorname{Im} t_{m} \geqq-\beta$. The value of (3.28) at the imaginary points $t_{k}=-i s_{k}, k=1, \ldots, m$ and $0=s_{0} \leqq s_{1} \cdots \leqq s_{m}=\beta$ is by (3.26) given by

$$
\omega_{\beta}^{0}(\Lambda)\left(F_{0} \alpha_{-i s_{1}}^{0}(\Lambda)\left(F_{1}\right) \ldots \alpha_{-i s_{m}}^{0}(\Lambda)\left(F_{m}\right)\right)=E_{\Lambda}^{\beta}\left[\prod_{k=0}^{m} F_{k}^{s_{k}}\right],
$$

where $F_{k}^{s_{k}}$ is the translated by an amount $s^{k}$ around the circle $S_{\beta}$ of the functions $F_{k}$ in $\mathscr{F}(\mathcal{O})$. Since $F_{k}, k=0, \ldots, n$ are continuous bounded functions of the stochastic variables $\left(\eta, \varphi_{1}\right), \ldots,\left(\eta, \varphi_{n}\right)$, we get that (3.29) converge if the corresponding correlation function converge since $E_{A}^{\beta}$ is the expectation with respect to a Gaussian distribution. We shall now assume that the mass $m>0$. By Theorem 3.1 the correlation function for $E_{\Lambda}^{\beta}$ is given by $G_{\Lambda}^{\beta}(x, y, s-t)$. That $G_{\Lambda}^{\beta}(x, y, s-t)$ converges as $\Lambda$ tends to $R^{n}$ in such a way that it finally contains all bounded sets follows from the fact that $G_{A}^{\beta}$ is the Greens function for the self adjoint operator

$$
-\frac{\partial^{2}}{\partial t^{2}}-\sum_{i=1}^{n} \frac{\partial^{2}}{\partial x_{i}^{2}}+m^{2}
$$

on $S_{\beta} \times \Lambda$, with some self adjoint boundary conditions on $\partial \Lambda$. So that as $\Lambda$ tends to $R^{n}$ in such a way as to finally containing any bounded set we get that $G_{A}^{\beta}(x, y, s-t)$ converge weakly to $G^{\beta}(x, y, s-t)$ which is the Greens function on $S_{\beta} \times R^{n}$ for the operator (3.30).

Since the local algebra for the free field $\mathscr{A}_{0}$ is the smallest norm closed $C^{*}$-algebra containing $\alpha_{t}^{0}(F)$ for all $t$ and $F \in \mathscr{F}(\mathcal{O})$ for some $\mathcal{O}$, we have that elements of the form $F_{0} \alpha_{t_{1}}^{0}\left(F_{1}\right) \ldots \alpha_{t_{m}}^{0}\left(F_{m}\right)$ are norm dense in $\mathscr{A}_{0}$. Hence we have proved the following theorem.

Theorem 3.2. Let $\mathscr{A}_{0}$ be the local algebra for the free field, then $\alpha_{t}^{0}$ defines a group of $C^{*}$-automorphism of $\mathscr{A}_{0}$. There is a state $\omega_{\beta}^{0}$ on $\mathscr{A}_{0}$ which is invariant under $\alpha_{t}^{0}$ i.e.

$$
\omega_{\beta}^{0}\left(B \cdot \alpha_{t}^{0}(C)\right)=\omega_{\beta}^{0}\left(\alpha_{-t}^{0}(B) \cdot C\right),
$$


such that $\omega_{\beta}^{0}\left(B \alpha_{t}^{0}(C)\right)$ is analytic in the strip $-\beta<\operatorname{Im} t<0$ and uniformly bounded and continuous in $-\beta \leqq \operatorname{Im} t \leqq 0$, and satisfies the KMS conditions on the boundary

$$
\omega_{\beta}^{0}\left(B \cdot \alpha_{t-i \beta}^{0}(C)\right)=\omega_{\beta}^{0}\left(C \cdot \alpha_{-t}^{0}(B)\right)
$$

for real $t$.

Moreover, if $F_{0}, \ldots, F_{m}$ is in the subalgebra of $\mathscr{A}_{0}$ generated by the fields at time zero then $\omega_{\beta}^{0}\left(F_{0} \alpha_{t_{1}}^{0}\left(F_{1}\right) \ldots \alpha_{t_{m}}^{0}\left(F_{m}\right)\right)$ is analytic in $0>\operatorname{Im} t_{1}>\cdots>\operatorname{Im} t_{m}>-\beta$ and continuous and uniformly bounded in $0 \geqq \operatorname{Im} t_{1} \geqq \cdots \geqq-\beta$, and its value at the imaginary points $t_{k}=-i s_{k}$, $k=1, \ldots, m$ with $0=s_{0} \leqq s_{1} \cdots \leqq s_{m}=\beta$ is given by

$$
\omega_{\beta}^{0}\left(F_{0} \alpha_{-i s_{1}}^{0}\left(F_{1}\right) \ldots \alpha_{-i s_{m}}^{0}\left(F_{m}\right)\right)=E^{\beta}\left[\prod_{k=0}^{m} F_{k}^{s_{k}}\right],
$$

where $E^{\beta}$ is the expectation with respect to the generalized Gaussian process with mean zero and covariance function $F^{\beta}(x-y, s-t)$, which is the Greensfunction on $S_{\beta} \times R^{n}$ for the self adjoint operator

$$
-\frac{\partial^{2}}{\partial t^{2}}-\sum_{i=1}^{n} \frac{\partial^{2}}{\partial x_{i}^{2}}+m^{2}
$$

on $L_{2}\left(S_{\beta} \times R^{n}\right)$, and $F_{k}^{s_{k}}$ is the translated by the action of the circle group $S_{\beta}$ on $S_{\beta} \times R^{n}$ of the function $F_{k}$ by the amount $s_{k}$.

Furthermore, if $B \in \mathscr{A}_{0}$ is in $\overline{\mathscr{A}}(\mathcal{O})$ for some bounded $\mathcal{O}$ then

$$
\omega_{\beta}^{0}(B)=\lim _{A} \omega_{\beta}^{0}(\Lambda)(B)
$$

as $\Lambda$ tends to $R^{n}$ in the sense that $\Lambda$ finally contains any fixed bounded set.

Remark. Utilizing the formula (2.24) we get that $G^{\beta}(x, t)$ is given by

$$
G^{\beta}(x, s)=\frac{1}{(2 \pi)^{n}} \int_{R^{n}} \hat{G}^{\beta}(p, s) e^{-i p x} d p
$$

where for $0 \leqq s \leqq \beta$

$$
\hat{G}^{\beta}(p, s)=\left(2 \omega\left(1-e^{-\beta \omega}\right)\right)^{-1}\left(e^{-s \omega}+e^{-(\beta-s) \omega}\right)
$$

with $\omega=\omega(p)=\sqrt{p^{2}+m^{2}}$.

If we introduce the annihilation creation operators and the free fields we have the relations

$\varphi(x, t)=\frac{1}{\sqrt{2}}(2 \pi)^{-\frac{n}{2}} \int\left[e^{i(p x+\omega t)} a^{*}(p)+e^{-i(p x+\omega t)} a(p)\right] \frac{d p}{\omega(p)^{\frac{1}{2}}}$

where $\varphi(x, t)$ is the free field at time $t$. 
The operator that counts the number of particles with momentum $p \in \Omega$ in a region $\Omega \subset R^{n}$ of momentumspace is given by

$$
N(\Omega)=\int_{\Omega} a^{*}(p) a(p) d p
$$

Introducing now the function

so that

$$
\Delta^{\beta}(x, t)=G^{\beta}(x,-i t)
$$

$$
\hat{\Delta}^{\beta}(p, t)=\left(2 \omega\left(1-e^{-\beta \omega}\right)\right)^{-1}\left(e^{i t \omega}+e^{-\beta \omega} e^{-i t \omega}\right)
$$

we get the following formula for computing expectations of products of fields

$\omega_{\beta}^{0}\left(\varphi\left(x_{1}, t_{1}\right) \ldots \varphi\left(x_{n}, t_{n}\right)\right)=\left\{\begin{array}{l}\sum \Delta^{\beta}\left(x_{i_{1}}-x_{i_{2}}, t_{i_{1}}-t_{i_{2}}\right) \ldots \Delta^{\beta}\left(x_{i_{n-1}}-x_{i_{n}}, t_{i_{n-1}}-t_{i_{n}}\right) \\ \quad \text { for } n \text { even } \\ 0 \text { for } n \text { odd }\end{array}\right.$

where the summation runs over all partitions of $(1, \ldots, 2 k)$ with $2 k=n$ into disjoint pairs $\left(i_{1}, i_{2}\right)\left(i_{3}, i_{4}\right) \ldots\left(i_{n-1}, i_{n}\right)$.

If we define the pressure for the free field at temperature $\frac{1}{\beta}$ in the usual way by

$$
p_{\beta}^{0}=\beta^{-1} \lim _{\Lambda \rightarrow R^{n}}|\Lambda|^{-1} \log \left(\operatorname{tr}\left(e^{-\beta H_{0}(\Lambda)}\right)\right),
$$

where $|\Lambda|$ is the volume of $\Lambda$, we get by using the formuly

$$
\operatorname{tr}\left(e^{-\beta H_{0}(A)}\right)=\left|1-e^{-\beta A_{\Lambda}}\right|^{-1}
$$

together with well known asymptotic formulas for the eigenvalues of the Laplacian $\Delta$ in $\Lambda$ as $\Lambda$ increase to $R^{n}$, we get that the limit (3.39) always exists and is given by

$$
p_{\beta}^{0}=-(2 \pi)^{-n} \beta^{-1} \int_{R^{n}} \log \left(1-e^{-\beta \omega(p)}\right) d p .
$$

If we take $\Lambda_{l}=\left[-\frac{l}{2}, \frac{l}{2}\right]^{n}$ with periodic boundary conditions we have that $A_{A_{l}}$ has the eigenvalues

$$
\left(\sum_{i=1}^{n}\left(\frac{2 \pi n_{i}}{l}\right)^{2}+m^{2}\right)^{\frac{1}{2}}
$$

where $\left(n_{1}, \ldots, n_{n}\right) \in \mathbb{Z}^{n}$. In this case we have the annihilation creation operators $a_{l}^{*}(p)$ and $a_{l}(p)$ with $p \in \frac{2 \pi}{l} \cdot \mathbb{Z}^{n}$, for $H_{0}\left(\Lambda_{l}\right)$. The operator that counts the number of particles with momentum $p \in \Omega$ in a region $\Omega \subset R^{n}$ is now given by

$$
N_{l}(\Omega)=\sum_{p \in \Omega} a_{l}^{*}(p) a_{l}(p)
$$


If we now compute the expected number of particles for the system in $\Lambda_{l}$ we get

$$
\omega_{\beta}^{0}\left(\Lambda_{l}\right)\left(N_{l}(\Omega)\right)=\sum_{p \in \Omega} \frac{e^{-\beta \omega(p)}}{1-e^{-\beta \omega(p)}} .
$$

We now define the density of particles with momentum in $\Omega$ by

$$
\int \varrho_{\beta}^{0}(p) d p=\lim _{\Lambda_{1} \rightarrow R^{n}}\left|\Lambda_{l}\right|^{-1} \omega_{\beta}^{0}\left(\Lambda_{l}\right)\left(N_{l}(\Omega)\right) .
$$

Then this limit exists and is given by

$$
\int_{\Omega} \varrho_{\beta}^{0}(p) d p=(2 \pi)^{-n} \int_{\Omega} \frac{e^{-\beta \omega(p)}}{1-e^{-\beta \omega(p)}} d p .
$$

So that then density of particles with momentum $p$ exists and is given by

$$
\varrho_{\beta}^{0}(p)=(2 \pi)^{-n} \frac{e^{-\beta \omega(p)}}{1-e^{-\beta \omega(p)}},
$$

and the particle density is given by

$$
\varrho_{\beta}^{0}=(2 \pi)^{-n} \int_{R^{n}} \frac{e^{-\beta \omega(p)}}{1-e^{-\beta \omega(p)}} d p .
$$

In correspondence with (3.45) and (3.46) we may introduce the partial pressure due to particles with momentum $p$ by

$$
p_{\beta}^{0}(p)=-(2 \pi)^{-n} \beta^{-1} \log \left(1-e^{-\beta \omega(p)}\right) .
$$

If we want to express the state $\omega_{\beta}^{0}$ in terms of annihilation creation operators

$$
a^{\#}(h)=\int h(p) a^{\#}(p) d p
$$

where $a^{\#}$ stands for a or $a^{*}$, and $h \in L_{2}\left(R^{n}\right)$, then we have the formula

$$
\omega_{\beta}^{0}\left(e^{i\left(a^{*}(\bar{h})+a(h)\right)}\right)=e^{-\frac{1}{2} \int|h(p)|^{2} \frac{1+e^{-\beta \omega(p)}}{1-e^{-\beta \omega(p)}}}
$$

As a comparison we have that the corresponding quantity for a system of free Schrödinger particles at temperature $\beta$ and activity $z$ is given by

$$
e^{-\frac{1}{2} \int|h(p)|^{2}} \frac{1+z e^{-\frac{\beta}{2 m} p^{2}}}{1-z e^{-\frac{\beta}{2 m} p^{2}}} d p
$$


To within the non relativistic approximation

$$
\omega(p) \sim m+\frac{1}{2 m} p^{2}
$$

we see that $\omega_{\beta}^{0}$ is the Gibbs state of free Schrödinger particles of mass $m$ at temperature $\beta$ and activity $z=e^{-m \beta}$.

\section{The Gibbs-State for the Interacting Scalar Quantum Field in Two Space-Time Dimensions}

In the case of two space-time dimensions or equivalently one space dimension, the interacting scalar field is relatively well understood in the case of polynomial interactions $([2,7-10])$ and exponential interactions ([3] and [11]). In the case of positive mass $m>0$, it was proved by Glimm-Spencer [2] that the thermodynamic limit for the temperature zero $(\beta=\infty)$ state existed and is unique for weak polynomial interactions. More recently Nelson [12] has established the existence of the thermodynamic limit for strong polynomial interactions with Dirichlet boundary conditions. Nelson's method which depends strongly on the Dirichlet boundary conditions leads to the question of whether this limit is unique, and in fact Dobrushin and Minlos [6] have announced the result that there is a certain critical value for the interaction strength for any even polynomial interaction above which the limit is not unique. For the strong exponential interaction the existence and uniqueness of the termodynamic limit for the temperature zero state was recently proved by Albeverio and Høegh-Krohn [3], in the case of even interactions.

From what is said above we see that the thermodynamic behavior of the temperature zero state is quite complex and that by the result of Dobrushin and Minlos there are phasetransitions of the temperature zero state for the even polynomial interactions.

In contrast with this complex picture for the temperature zero state, we shall see that for the positive temperature $(\beta<\infty)$ state or the Gibbs state the thermodynamic limit always exists and is unique for the polynomial interactions as well as for the exponential interactions, without any restriction on the strength of the interaction, in the case of two space-time dimensions.

Now let

$$
H_{l}=H_{0}+\int_{-l}^{l}: V(\varphi(x)): d x
$$

where $\varphi(x)$ is the time zero free field of positive mass $m>0$, in two space time dimensions, and $V(s)$ is either a polynomial which is bounded below

$$
V(s)=P(s)
$$


or an exponential function i.e.

$$
V(s)=\int e^{\alpha s} d \mu(\alpha)
$$

where $d \mu$ is a positive measure of compact support in the open intervall $(-\sqrt{2 \pi}, \sqrt{2 \pi})$.

$H_{0}$ is the Hamiltonian for the free scalar field $\varphi . H_{l}$ is then the Hamiltonian for the corresponding interacting field with a space cut off interaction. For details concerning the definition of $H_{l}$ the reader should consult the Refs. [7] and [10] for the case (4.2) and the Ref. [11] for the case (4.3).

It is known that $H_{l}$ is essentially self adjoint on the intersection of the domains of $H_{0}$ and $V_{l}$

$$
V_{l}=\int_{-l}^{l}: V(\varphi(x)): d x
$$

and that $H_{l}$ is bounded below

$$
H_{l} \geqq-b
$$

where $b$ is some real number depending on $V_{l}$, so that $e^{-\beta H_{l}}$ is a bounded operator.

We will now construct the Gibbs state for the space cut-off interaction (4.1).

Let $\Lambda$ be an interval containing the interval $[-l, l]$ in its interior, then we set

$$
H_{l}(\Lambda)=H_{0}(\Lambda)+\int_{-l}^{l}: V(\varphi(x)): d x
$$

By the same methods that proves that $H_{l}$ is essentially self adjoint and bounded below we get that $H_{l}(\Lambda)$ is essentially self adjoint and bounded below. Moreover, we also get that $H_{l}(\Lambda)$ has discrete spectrum and that $e^{-\beta H_{l}(\Lambda)}$ is of trace class. We shall start by computing the trace of $e^{-\beta H_{l}(A)}$. By the method of hypercontractivity [13] in the same way as for $H_{l}$, we have that $H_{l}(\Lambda)$ may be approximated by operators $H_{n}$ such that

$$
\left\|e^{-\beta H_{l}(\Lambda)}-e^{-\beta H_{n}}\right\| \rightarrow 0
$$

and $H_{n}$ has the form

$$
H_{l}(\Lambda)=H_{0}(\Lambda)+V_{l}^{(n)}
$$

where $V_{l}^{(n)}$ is in $\mathscr{F}(\mathcal{O})$ for some $\mathcal{O} \subset \Lambda$. We shall prove below that

$$
\operatorname{tr} e^{-\beta H_{n}} \rightarrow \operatorname{tr} e^{-\beta H_{l}(\Lambda)} .
$$


Since $V_{l}^{(n)}$ is bounded we get by the Trotter-Kato product formula that

$$
\text { strong } \lim _{k \rightarrow \infty}\left[e^{-\frac{\beta}{k} H_{0}(\Lambda)} e^{\left.-\frac{\beta}{k} V_{l}^{(n)}\right]^{k}}=e^{-\beta H_{n}},\right.
$$

but this may obviously also be written in the form

$$
\text { strong } \lim _{k \rightarrow \infty}\left[e^{-\frac{\beta}{2 k} H_{0}(\Lambda)} e^{-\frac{\beta}{k} V_{l}^{(n)}} e^{-\frac{\beta}{2 k} H_{0}(\Lambda)}\right]^{k}=e^{-\beta H_{n}} .
$$

Now let $V_{l}^{(n)} \geqq-c$, where $c$ of course depends on $n$. Then

hence

$$
\left\|e^{-\frac{\beta}{k} V_{l}^{(n)}}\right\| \leqq e^{\frac{\beta}{k} c}
$$

$$
e^{-\frac{\beta}{2 k} H_{0}(\Lambda)} e^{-\frac{\beta}{k} V_{l}^{(n)}} e^{-\frac{\beta}{2 k} H_{0}(\Lambda)} \leqq e^{\frac{\beta}{k} c} e^{-\frac{\beta}{k} H_{0}(\Lambda)},
$$

so that the $i$-th eigenvalue of

$$
\left[e^{-\frac{\beta}{2 k} H_{0}(\Lambda)} e^{-\frac{\beta}{k} V_{l}^{(n)}} e^{-\frac{\beta}{2 k} H_{0}(\Lambda)}\right]^{k}
$$

is smaller or equal to $e^{\beta c}$ times the $i$-th eigenvalue of $e^{-\beta H_{0}(A)}$. On the other hand we have by (4.11) that the $i$-th eigenvalue of (4.14) converge to the $i$-th eigenvalue of $e^{-\beta H_{n}}$. Hence by dominated convergence we get that the trace of (4.14) converge to the trace of $e^{-\beta H_{n}}$, since $e^{-\beta H_{0}(\Lambda)}$ is of trace class.

However the trace of (4.14) is by Theorem 3.1 given by

$$
\left|1-e^{-\beta A_{\Lambda}}\right|^{-1} E_{\Lambda}^{\beta}\left[\exp \left\{-\frac{\beta}{k} \sum_{j=1} V_{l}^{(n)}\left(\eta\left(\frac{\beta}{k}\left(j-\frac{1}{2}\right)\right)\right)\right\}\right] .
$$

Since the transformation $V_{l}^{(n)}(\eta(0))-V_{l}^{(n)}(\eta(s))$ is induced by the action of the circle group $S_{\beta}$ on $S_{\beta} \times \Lambda$, and the generalized Gaussian process corresponding to $E_{\Lambda}^{\beta}$ is homogeneous with respect to this action, we have that the transformation is given by a strongly continuous unitary group on $L_{2}$ of the corresponding process, and therefore $V_{l}^{(n)}(\eta(s))$ is a strongly continuous function of $s$ in the $L_{2}$ space of the process. Hence we get the strong $L_{2}$-convergence

$$
\frac{\beta}{k} \sum_{j=1}^{k} V_{l}^{(n)}\left(\eta\left(\frac{\beta}{k}\left(j-\frac{1}{2}\right)\right)\right) \rightarrow \int_{0}^{\beta} V_{l}^{(n)}(\eta(s)) d s
$$

as $k \rightarrow \infty$. By passing to an almost everywhere convergent subsequence, we get by dominated convergence the corresponding convergence of (4.15). Hence we have that

$$
\operatorname{tr} e^{-\beta H_{n}}=\left|1-e^{-\beta A_{\Lambda}}\right|^{-1} E_{\Lambda}^{\beta}\left[e^{-\int_{0}^{\beta} V_{l}^{(n)}(\eta(s)) d s}\right] .
$$


Now the approximation of $V_{l}=\int_{-l}^{l}: V(\varphi(x)): d x$ by functions $V_{l}^{(n)}$ in $\mathscr{F}(\mathcal{O})$ may be carried out in two steps. First we approximate $V_{l}^{[a, b]}$, where $V_{l}^{[a, b]}$ is equal to $V_{l}$ in those points where $V_{l}$ has values in the interval $[a, b]$ and $V_{l}^{[a, b]}$ is equal to zero elsewhere. Under this approximation we have that $V_{l}^{(n)}$ is uniformly bounded below so that $e^{-\beta H_{n}}$ $\leqq e^{c \beta} \cdot e^{-\beta H_{0}(\Lambda)}$, and by hypercontractivity $e^{-\beta H_{n}}$ converge to $e^{-\beta\left(H_{0}(\Lambda)+V_{l}^{[a, b])}\right.}$ in norm, so that $\operatorname{tr} e^{-\beta H_{n}}$ converge by dominated convergence. On the other hand the right hand side of (4.17) will also converge by dominated convergence since under this approximation $V_{l}^{(n)}$ is uniformly bounded below.

Then we remove $a$ and $b$ by first letting $a \rightarrow-\infty$ and then $b \rightarrow \infty$. In both cases we have that both sides of the equation

$$
\operatorname{tr} e^{-\beta\left(H_{0}(A)+V_{l}^{[a, b]}\right)}=\left|1-e^{-\beta A_{\Lambda}}\right|^{-1} E_{\Lambda}^{\beta}\left[e^{-\int_{0}^{\beta} V_{l}^{[a, b]}(\eta(s)) d s}\right]
$$

converge by monotone convergence. Hence we have proved the following formula

$$
\operatorname{tr} e^{-\beta H_{l}(\Lambda)}=\left|1-e^{-\beta A_{\Lambda}}\right|^{-1} E_{\Lambda}^{\beta}\left[e^{-\int_{0}^{\beta} V_{l}(\eta(s)) d s}\right]
$$

Recalling the form of $V_{l}$ this may also be written

$$
\operatorname{tr} e^{-\beta H_{l}(\Lambda)}=\left|1-e^{-\beta A_{\Lambda}}\right|^{-1} E_{\Lambda}^{\beta}\left[e^{-\int_{0}^{\beta} \int_{-l}^{l}: V(\xi(x, s)): d x d s}\right] .
$$

In the same way as we proved the formula (4.20) we prove the following

Lemma 4.1. Let $F_{0}, \ldots, F_{n-1}$ be in $L_{\infty}\left(h_{A}\right)$ and $0=s_{0} \leqq s_{1} \leqq \cdots \leqq s_{n}=\delta$ then

$$
\begin{aligned}
\operatorname{tr}\left(F_{0} e^{-s_{1} H_{l}(\Lambda)} F_{1} e^{-\left(s_{2}-s_{1}\right) H_{l}(\Lambda)} \ldots F_{n-1} e^{-\left(\beta-s_{n}\right) H_{l}(\Lambda)}\right) & \\
& =\left|1-e^{-\beta A_{\Lambda}}\right|^{-1} E_{\Lambda}^{\beta}\left[\prod_{k=0}^{n-1} F_{k}^{s_{k}} e^{-\int_{0}^{\beta} \int_{-i}^{l}: V(\zeta(x, s)): d x d s}\right]
\end{aligned}
$$

where $F_{k}^{s_{k}}$ is the translation of $F_{k}$ by the amount $s_{k}$ in the action induced by the circle group $S_{\beta}$ on the generalized Gaussian process $\xi(x, s)$.

As in Section 3 we now define for any $B \in B\left(L_{2}\left(h_{A}\right)\right)$

and

$$
\begin{gathered}
\alpha_{t}^{l}(\Lambda)(B)=e^{-i t H_{l}(\Lambda)} B e^{i t H_{l}(\Lambda)} \\
\omega_{\beta}^{l}(\Lambda)(B)=\left(\operatorname{tr} e^{-\beta H_{l}(\Lambda)}\right)^{-1} \operatorname{tr}\left(B e^{-\beta H_{l}(\Lambda)}\right) .
\end{gathered}
$$

We then have that if $B \in \mathscr{A}(\mathcal{O})$ and $\mathcal{O}_{t} \subset A$, then $\alpha_{t}^{l}(\Lambda)$ is independent of $\Lambda$, and we denote this $\Lambda$ independent value by $\alpha_{t}^{l}(B)$. This then gives us a 
group of $C^{*}$-automorphism $\alpha_{t}^{l}$ on $\overline{\mathscr{A}}$. It is well known that if $\mathcal{O}_{t} C[-l, l]$ then $\alpha_{t}^{l}(B)$ is independent of $l$, and we shall denote this $l$ independent value by $\alpha_{t}(B)$, and again $\alpha_{t}$ gives us a group of $C^{*}$-automorphism on $\mathscr{A}$.

Let now $\mathscr{A}$ be the smallest norm closed $C^{*}$-algebra in $\overline{\mathscr{A}}$ containing $\alpha_{t}(F)$ for all real $t$ and all $F \in \mathscr{F}(\mathcal{O})$ for any bounded $\mathcal{O}$ in $R$. Elements in $\mathscr{A}$ of the form

$$
F_{0} \alpha_{t_{1}}\left(F_{1}\right) \ldots \alpha_{t_{n}}\left(F_{n}\right)
$$

with $F_{0}, F_{1}, \ldots, F_{n}$ in $\mathscr{F}(\mathcal{O})$ then span a dense linear set in $\mathscr{A}$. We shall see that

$$
\omega_{\beta}^{L}(\Lambda)\left(F_{0} \alpha_{t_{1}}\left(F_{1}\right) \ldots \alpha_{t_{n}}\left(F_{n}\right)\right)
$$

converges as $A$ tends to $R$ and $l$ tends to $\infty$.

If $\mathcal{O}_{t_{i}} \subset[-l, l] \subset A i=1, \ldots, n$ then (4.23) is equal to

$$
\omega_{\beta}^{l}(\Lambda)\left(F_{0} \alpha_{t_{1}}^{l}(\Lambda)\left(F_{1}\right) \ldots \alpha_{t_{n}}^{l}(\Lambda)\left(F_{n}\right)\right) .
$$

By the definition (4.22) of $\omega_{\beta}^{l}(\Lambda)$ we have that (4.25) is analytic in $0>\operatorname{Im} t_{1}>\cdots>\operatorname{Im} t_{n}>-\beta$ and uniformly bounded and continuous in $0 \geqq \operatorname{Im} t_{1} \geqq \cdots \geqq \operatorname{Im} t_{n} \geqq-\beta$. Moreover, its values at the imaginary points $t_{k}=-i s_{k} \quad k=1, \ldots, n$ with $0=s_{0} \leqq s_{1} \cdots \leqq s_{n}$ is by Lemma 4.1 given by

$$
\begin{aligned}
& \left.\omega_{\beta}^{l}(\Lambda) F_{0} \alpha_{-i s_{1}}^{l}(\Lambda)\left(F_{1}\right) \ldots \alpha_{-i s_{n}}^{l}(\Lambda)\left(F_{n}\right)\right)
\end{aligned}
$$

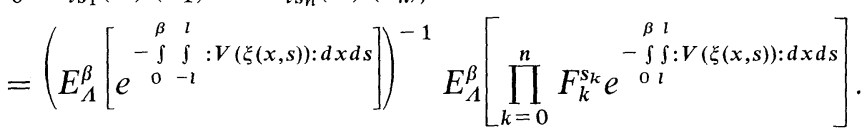

To prove that (4.24) converges as first $\Lambda$ tends to $R$ and then $l$ tends to $\infty$, it is therefore enough to prove that the same limits exists for the right hand side of (4.26). Since $F_{k} \in \mathscr{F}(\mathcal{O}) k=0, \ldots, n$, it is therefore enough; if we want to prove that the limit exists as $\Lambda \rightarrow R$, to prove that $E_{\Lambda}^{\beta}$ converge weakly as $\Lambda \rightarrow R$. But since $E_{\Lambda}^{\beta}$ is the expectation with respect to the generalized Gaussian process with mean zero and covariance function $G_{\Lambda}^{\beta}(x, y ; s-t)$, the weak convergence of $E_{A}^{\beta}$ follows from that of the covariance function. Hence we find that the limit of (4.26) as $\Lambda \rightarrow R$ exists and is given by

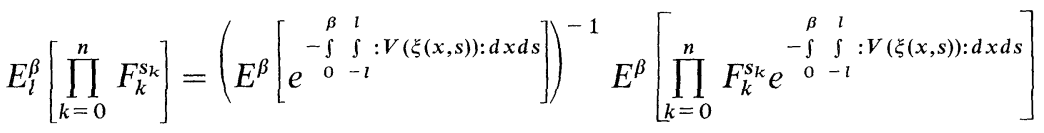

where $E^{\beta}$ is the expectation with respect to the generalized Gaussian process with mean zero and covariance given by the Greensfunction 
$G^{\beta}(x-y, s-t)$ for the self adjoint operator

$$
-\frac{\partial^{2}}{\partial t^{2}}-\frac{\partial^{2}}{\partial x^{2}}+m^{2}
$$

on $S_{\beta} \times R$. For $0 \leqq t \leqq \beta$ we have that $G^{\beta}(x, t)$ is given by

where

$$
G^{\beta}(x, t)=\frac{1}{2 \pi} \int_{R} e^{-i x p} \hat{G}^{\beta}(p, t) d p
$$

$$
\hat{G}^{\beta}(p, t)=\left[2 \omega(p)\left(1-e^{-\beta \omega(p)}\right)\right]^{-1}\left[e^{-t \omega(p)}+e^{-(\beta-t) \omega(p)}\right]
$$

where $\omega(p)=\sqrt{p^{2}+m^{2}}$.

To prove that (4.27) converges as $l \rightarrow \infty$ it is again enough, since $F_{k} \in \mathscr{F}(\mathcal{O})$ for $k=0, \ldots, n$, to prove that $E_{l}^{\beta}$ converges weakly as $l \rightarrow \infty$. To do this we consider the Fourier transform of the generalized process given by $E_{l}^{\beta}$

$$
E_{l}^{\beta}\left[e^{i \iint \psi(x, s) \xi(x, s) d x d s}\right]
$$

where $\psi$ is a $C^{\infty}$-function of compact support in $S_{\beta} \times R$. By (4.27) we have that (4.31) is given by

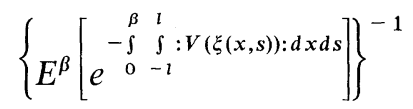

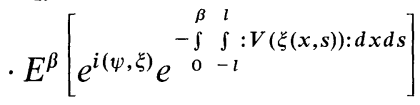

Let $\psi$ have support in $S_{\beta} \times[-a, a]$.

Consider now the Hamiltonian $H_{\beta}$ on $L_{2}\left(h_{\beta}\right)$ where $h_{\beta}=h_{\Lambda_{\beta}}$ with $\Lambda_{\beta}=[0, \beta]$ with periodic boundary conditions and $H_{\beta}$ is the corresponding periodic Hamiltonian

$$
H_{\beta}=H_{0}^{\beta}+\int_{0}^{\beta}: V(\varphi(x)): d x
$$

where $H_{0}^{\beta}=H_{0}\left(\Lambda_{\beta}\right), \Lambda_{\beta}=[0, \beta]$ with periodic boundary conditions. It is well known both in the polynomial and exponential case that $H_{\beta}$ has a simple lowest eigenvalue with a normalized eigenvector which we denote $\Omega_{\beta}$.

By letting in Lemma $4.1 \beta$ tend to infinity and taking $\Lambda$ in Lemma 4.1 fixed equal to the $\Lambda_{\beta}$ above, we obtain easily the formulas

and

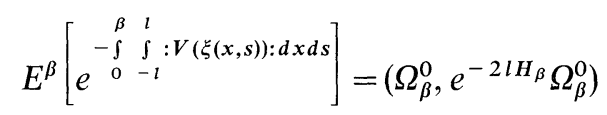

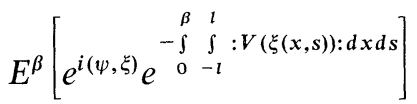

$$
\begin{aligned}
& =\left(e^{-(l-a) H_{\beta}} \Omega_{\beta}^{0}, W_{[-a, a]}(i f) e^{-(l-a) H_{\beta}} \Omega_{\beta}^{0}\right),
\end{aligned}
$$


where $\Omega_{\beta}^{0}$ is the normalized eigenvector of $H_{0}^{\beta}$ corresponding to it simple lowest eigenvalue and $W_{[s, t]}(i f)$ is the unique bounded operator satisfying the strong differential equation

$$
\frac{d}{d t} W_{[s, t]}(i f)=W_{[s, t]}(i f)\left[-H_{\beta}+i \int_{0}^{\beta} f(x, t) \varphi_{\beta}(x) d x\right]
$$

where $\varphi_{\beta}(x)$ is the free field corresponding to $H_{0}^{\beta}$, with the initial condition that

and

$$
\begin{gathered}
W_{[s, s]}(i f)=1 \\
f(x, t)=\psi(t, x) .
\end{gathered}
$$

The analog of (4.34) and (4.35) in the temperature zero case is well known and used for instance in [14] where they are called the Nelson symmetries.

Now that the limit of (4.31) and hence of (4.27) exists as $l \rightarrow \infty$ follows simply from the fact that $H_{\beta}$ has a simple lowest eigenvalue. Hence we have

Lemma 4.2. Let

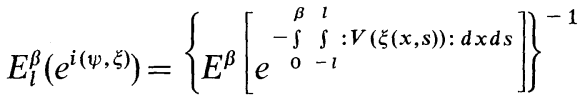

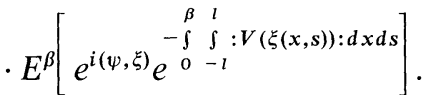

Then the limit $E_{\infty}^{\beta}\left(e^{i(\psi, \xi)}\right)$ as $l \rightarrow \infty$ exists and is given by

$$
E_{\infty}^{\beta}\left(e^{i(\psi, \xi)}\right)=\left(\Omega_{\beta}, W_{[-a, a]}(i f) \Omega_{\beta}\right)
$$

where $f(x, t)=\psi(t, x)$. Moreover, the measure induced by $E_{\infty}^{\beta}$ is locally equivalent to the generalized Gaussian process given by $E^{\beta}$, i.e. restricted to the subalgebras generated by $(\psi, \xi)$ for $\psi$ with support in a fixed interval $S_{\beta} \times[-a, a]$ they are equivalent. The measure given by $E_{\infty}^{\beta}$ is strongly mixing with respect to space translations i.e.

$$
\lim _{x \rightarrow \infty} E_{\infty}^{\beta}\left(e^{i\left(\psi_{1}, \xi\right)} e^{i\left(\psi_{2}^{x}, \xi\right)}\right)=E_{\infty}^{\beta}\left(e^{i\left(\psi_{1}, \xi\right)}\right) \cdot E_{\infty}^{\beta}\left(e^{i\left(\psi_{2}, \xi\right)}\right)
$$

where $\psi^{x}(t, y)=\psi(t, y-x)$.

Proof. We have already proved everything up to the moreover part. The moreover part follows immediately from the formula for $E_{\infty}^{\beta}$ and the strongly mixing follows from the same formula together with the fact that $\Omega_{\beta}$ belongs to a simple lowest eigenvalue of $H_{\beta}$.

Theorem 4.1. Let $\mathscr{A}$ be the local algebra for the interacting field, i.e. the smallest norm closed $C^{*}$-algebra in $\bar{A}$ containing $\alpha_{t}(F)$ for all real $t$ and all $F \in \mathscr{F}(\mathcal{O})$ for any bounded $\mathcal{O}$ in $R$. There exists then a state $\omega_{\beta}$ on $\mathscr{A}$ 
such that $\omega_{\beta}$ is invariant under $\alpha_{t}$ i.e.

$$
\omega_{\beta}\left(B \alpha_{t}(C)\right)=\omega_{\beta}\left(\alpha_{-t}(B) \cdot C\right),
$$

for any $B$ and $C$ in $\mathscr{A} \cdot \omega_{\beta}\left(B \cdot \alpha_{t}(C)\right)$ is analytic in the strip $-\beta<\operatorname{Im} t<0$ and uniformly bounded and continuous in $-\beta \leqq \operatorname{Im} t \leqq 0$, and satisfies the KMS conditions on the boundary

$$
\omega_{\beta}\left(B \cdot \alpha_{t-i \beta}(C)\right)=\omega_{\beta}\left(C \cdot \alpha_{-t}(B)\right)
$$

for real t. $\omega_{\beta}$ is invariant under space translations

$$
\omega_{\beta}\left(B_{x}\right)=\omega_{\beta}(B)
$$

and have the cluster property

$$
\lim _{x \rightarrow \infty} \omega_{\beta}\left(B_{x} \cdot C\right)=\omega_{\beta}(B) \cdot \omega_{\beta}(C) .
$$

$\omega_{\beta}$ is locally Fock, i.e. if we restrict $\omega_{\beta}$ to the subalgebra generated by $\alpha_{t}(F)$ for $t$ in a fixed interval $[-a, a]$ and $F \in \mathscr{F}(\mathcal{O})$. For a fixed bounded $\mathcal{O}$, then on this subalgebra $\omega_{\beta}$ induces the free Fock representation.

Moreover, if $F_{0}, \ldots, F_{n}$ is in $\mathscr{F}(\mathcal{O})$ for some bounded $\mathcal{O}$ then $\omega_{\beta}\left(F_{0} \alpha_{t_{1}}\left(F_{1}\right) \ldots \alpha_{t_{n}}\left(F_{n}\right)\right)$ is analytic in $0>\operatorname{Im} t_{1}>\cdots>\operatorname{Im} t_{n}>-\beta$ and continuous and uniformly bounded in $0 \geqq \operatorname{Im} t_{1} \geqq \cdots \geqq \operatorname{Im} t_{n} \geqq-\beta$, and its value at the imaginary points $t_{k}=-i s_{k}, k=1, \ldots, n$ with $0=s_{0} \leqq s_{1}$ $\leqq \cdots \leqq s_{n}=\beta$ is given by

$$
\omega_{\beta}\left(F_{0} \alpha_{-i s_{1}}\left(F_{1}\right) \ldots \alpha_{-i s_{n}}\left(F_{n}\right)\right)=E_{\infty}^{\beta}\left[\prod_{k=0}^{n} F_{k}^{s_{k}}\right],
$$

where $E_{\infty}^{\beta}$ is the expectation with respect to the generalized homogeneous process on $S_{\beta} \times R$ given in Lemma 4.2, and $F_{k}^{s_{k}}$ is the translated of the function $F_{k}$ by the amount $s_{k}$ in the action of the circle group $S_{\beta}$ on $S_{\beta} \times R$.

Furthermore, if $B$ is in the subalgebra generated by $\alpha_{t}(F)$ for $t$ in a fixed interval $[-a, a]$ and $F \in \mathscr{F}(\mathcal{O})$ for a fixed bounded $\mathcal{O}$ then

$$
\omega_{\beta}(B)=\lim _{l \rightarrow \infty} \lim _{\Lambda \rightarrow R} \omega_{\beta}^{l}(\Lambda)(B) .
$$

Proof. Linear combinations of elements of the form $F_{0} \alpha_{t_{1}}\left(F_{1}\right) \ldots \alpha_{t_{n}}\left(F_{n}\right)$ are norm dense in $\mathscr{A}$. We may show in the same way as for the temperature zero case that $\omega_{\beta}^{l}(\Lambda)$ is locally Fock uniformly in $l$ and $\Lambda$. Since for $l$ and $\Lambda$ big enough $\alpha_{t_{k}}\left(F_{k}\right)=\alpha_{t_{k}}^{l}(\Lambda)\left(F_{k}\right)$ and $\alpha_{t}^{l}(\Lambda)$ is strongly continuous in Fock space we therefore get that

$$
\omega_{\beta}^{l}(\Lambda)\left(F_{0} \alpha_{t_{1}}\left(F_{1}\right) \ldots \alpha_{t_{n}}\left(F_{n}\right)\right)
$$


when we restrict $t_{k}, k=1, \ldots, n$ to a bounded interval is continuous in $t_{k}, k=1, \ldots, n$ uniformly with respect to $l$ and $\Lambda$. By passing to subsequences we therefore get as first $\Lambda \rightarrow R$ and then $l \rightarrow \infty$ through subsequences that (4.39) has a limit

$$
\omega_{\beta}\left(F_{0} \alpha_{t_{1}}\left(F_{1}\right) \ldots \alpha_{t_{n}}\left(F_{n}\right)\right)
$$

which is continuous in $t_{k}, k=1, \ldots, n$. On the other hand we have already proved that (4.39) is analytic in $0>\operatorname{Im} t_{1}>\cdots>\operatorname{Im} t_{n}>-\beta$ and that at the imaginary points

$$
\omega_{\beta}^{l}(\Lambda)\left(F_{0} \alpha_{-i s_{1}}\left(F_{1}\right) \ldots \alpha_{-i s_{n}}\left(F_{n}\right)\right)
$$

with $0 \leqq s_{1} \leqq \cdots \leqq s_{n} \leqq \beta,(4.41)$ converges as first $\Lambda \rightarrow R$ and then $l \rightarrow \infty$.

If we denote the limit by $\omega_{\beta}\left(F_{0} \alpha_{-i s_{1}}\left(F_{1}\right) \ldots \alpha_{-i s_{n}}\left(F_{n}\right)\right)$ we get by Lemma 4.2 that

$$
\omega_{\beta}\left(F_{0} \alpha_{-i s_{1}}\left(F_{1}\right) \ldots \alpha_{-i s_{n}}(F)\right)=E_{\infty}^{\beta}\left(\prod_{k=0}^{n} F_{k}^{s_{k}}\right) .
$$

(4.42) being a limit of functions which are uniformly bounded and analytic in $0>\operatorname{Im} t_{1}>\cdots>\operatorname{Im} t_{n}>-\beta$ must itself be analytic and bounded in the same domain. Since (4.40) is the limit of boundary values of these functions, it must itself be the boundary value of (4.42). But this proves that (4.40) must be independent of the subsequences chosen, so that (4.39) converges as first $\Lambda \rightarrow R$ and then $l \rightarrow \infty$ to a limit (4.40) which is continuous in $t_{k}, k=1, \ldots, n$. Hence $(4.40)$ is the boundary value of a function which is analytic in $0>\operatorname{Im} t_{1}>\cdots>\operatorname{Im} t_{n}>-\beta$ and uniformly bounded and continuous in $0 \geqq \operatorname{Im} t_{1} \geqq \cdots \geqq \operatorname{Im} t_{n}>-\beta$ and its value at the imaginary points is given by (4.42).

Now $\omega_{\beta}$ as limit of states is again a state and extends by continuity to all of $\mathscr{A}$. The invariance under $\alpha_{t}$ follows from the corresponding invariance for $\omega_{\beta}^{l}(\Lambda)$ and similarily the $K M S$ condition. The translation invariance under space translations follows from (4.42) and the homogeneity of the generalized process given by $E_{\infty}^{\beta}$. This homogeneity follows from Lemma 4.2. The cluster property follows from the fact that $E_{\infty}^{\beta}$ is strongly mixing with respect to space translations. This proves the theorem.

Remark. If we now define the pressure $p_{\beta}(V)$ at the temperature $\frac{1}{\beta}$ for the interacting field by

$$
p_{\beta}(V)=\beta^{-1} \lim _{l \rightarrow \infty}\left|\Lambda_{l}\right|^{-1} \log \left(\operatorname{tr}\left(e^{-\beta H_{l}\left(\Lambda_{l}\right)}\right)\right)
$$


with $\Lambda_{l}=[-l, l]$, we get by Lemmas 4.1 and 4.2 and its proof that

$$
p_{\beta}(V)=p_{\beta}^{0}-\beta^{-1} e_{\beta}(V)
$$

where $V$ describes the interaction so that

$$
H_{l}\left(\Lambda_{l}\right)=H_{0}\left(\Lambda_{l}\right)+\int_{-l}^{l}: V(\varphi(x)): d x
$$

and $p_{\beta}^{0}$ is the pressure for the free field given by (3.38) and (3.41), and $e_{\beta}(V)$ is the lowest eigenvalue of the periodic Hamiltonian

$$
H_{\beta}=H_{0}^{\beta}+\int_{0}^{\beta}: V(\varphi(x)): d x
$$

where $H_{0}^{\beta}=H_{0}([0, \beta])$ with periodic boundary conditions.

We see that Theorem 4.1 gives a certain duality between the Gibbs state at temperature $1 / \beta$ for the infinite volume interaction and the corresponding vacuum or zero temperature state for the interaction in a periodic box of length $\beta$. We shall denote this duality by the duality principle for the relativistic Gibbs state. This duality principle may also be expressed in terms of the Wightman functions or if we want also in terms of the Schwinger functions for the interaction.

Let $\varphi(x, t)$ be the interacting field at time $t$, i.e.

$$
\varphi(x, t)=\alpha_{t}(\varphi(x))
$$

where $\varphi(x)$ is the field at time zero, and (4.46) is an equation between operator valued distributions in $x$ for fixed $t$. The Wightman functions at temperature $1 / \beta$ for the infinite volume interaction is given by

$$
W_{\infty}^{\beta}\left(x_{1}, t_{1}, \ldots, x_{n}, t_{n}\right)=\omega_{\beta}\left(\varphi\left(x_{1}, t_{1}\right) \ldots \varphi\left(x_{n}, t_{n}\right)\right),
$$

and the Wightman functions for the field in a periodic box of length $\beta$, at temperature zero $(\beta=\infty)$ is given by

$$
W_{\beta}\left(x_{1} t_{1}, \ldots, x_{n} t_{n}\right)=\left(\Omega_{\beta}, \varphi\left(x_{1}, t_{1}\right) \ldots \varphi\left(x_{n}, t_{n}\right) \Omega_{\beta}\right)
$$

where $\Omega_{\beta}$ is a normalized eigenvector belonging to the lowest eigenvalue $e_{\beta}(V)$ for the $H_{\beta}$, Hamiltonian in a periodix box of length $\beta$

$$
H_{\beta}=H_{0}^{\beta}+\int_{0}^{\beta}: V(\varphi(x)): d x
$$

where $V$ is either a polynomial which is bounded below or an exponential function of the type (4.3), and $H_{0}^{\beta}$ is the free Hamiltonian in a periodic box of length $\beta$. We have then that (4.47) is analytic in $t_{1}, \ldots, t_{n}$ in the domain $0>\operatorname{Im} t_{1}>\cdots>\operatorname{Im} t_{n}>-\beta$ and that (4.48) is analytic 
in $\operatorname{Im} t_{1}>\cdots>\operatorname{Im} t_{n}$. The values at the imaginary points $t_{k}=-i s_{k}$ for $0<s_{1}, \ldots<s_{n}<\beta$ for (4.47) and $s_{1}<\cdots<s_{n}$ for (4.48) is called the Schwinger functions

and

$$
S_{\infty}^{\beta}\left(x_{1}, s_{1}, \ldots, x_{n}, s_{n}\right)=W_{\infty}^{\beta}\left(x_{1},-i s_{1}, \ldots, x_{n},-i s_{n}\right)
$$

$$
S_{\beta}^{\infty}\left(x_{1}, s_{1}, \ldots, x_{n}, s_{n}\right)=W_{\beta}^{\infty}\left(x_{1},-i s_{1}, \ldots, x_{n},-i s_{n}\right) \text {. }
$$

We may now express the duality principle from Theorem 4.1 in terms of Wightman- and Schwinger functions, and this gives us the following duality theorem

Theorem 4.2. (The duality theorem). Let $W_{\infty}^{\beta}\left(x_{1}, t_{1} \ldots x_{n}, t_{n}\right)$ be the Wightman functions at temperature $1 / \beta$ for the infinite volume interaction, and let $W_{\beta}^{\infty}$ be the usual Wightman functions at temperature zero $(\beta=\infty)$ for the interacting field in a periodic box of length $\beta$. Let $S_{\infty}^{\beta}$ and $S_{\beta}^{\infty}$ be the corresponding Schwinger functions, i.e. the Wightman functions at imaginary time, so that $W_{\beta}^{\infty}\left(x_{1} t_{1}, \ldots, x_{n} t_{n}\right)$ and $S_{\beta}^{\infty}\left(x_{1} s_{1}, \ldots, x_{n} s_{n}\right)$ is periodic with period $\beta$ in $x_{1}, \ldots, x_{n}$.

Then $W_{\infty}^{\beta}\left(x_{1}, t_{1}, \ldots x_{n}, t_{n}\right)$ is analytic in $0>\operatorname{Im} t_{1}>\cdots>\operatorname{Im} t_{n}>-\beta$, and $W_{\beta}^{\infty}\left(x_{1}, t_{1}, \ldots x_{n}, t_{n}\right)$ is analytic in $\operatorname{Im} t_{1}>\cdots>\operatorname{Im} t_{n}$, and for the corresponding Schwinger functions we have

$$
S_{\infty}^{\beta}\left(x_{1}, s_{1}, \ldots, x_{n}, s_{n}\right)=S_{\beta}^{\infty}\left(s_{1}, x_{1}, \ldots, s_{n}, x_{n}\right) .
$$

Moreover the difference between the pressure for the free and the interacting field at temperature $1 / \beta$ is equal to

$$
p_{\beta}(0)-p_{\beta}(V)=\beta^{-1} e_{\beta}(V)
$$

where $e_{\beta}(V)$ is the lowest eigenvalue for the interacting Hamiltonian in the periodic box of length $\beta$.

Remark. It is understood that the interactionterms in the Hamiltonians for the dual Wightman functions $W_{\infty}^{\beta}$ and $W_{\beta}^{\infty}$ are defined by consistent Wick orderings, i.e. if the Wick ordering for the interaction of $W_{\infty}^{\beta}$ is with respect to the free vacuum respectively the free bubbs state, then the Wick ordering for the interaction of $W_{\beta}^{\infty}$ is with respect to the vacuum for $H_{0}$ respectively the vacuum for $H_{\beta}$.

\section{References}

1. Ruelle, D.: Statistical mechanics. Rigorous results. New York: Benjamin 1969

2. Glimm, J., Jaffe, A., Spencer, T.: The Wightman axioms and particle structure of the $P(\varphi)_{2}$ quantum field model. Ann. Math. (To appear)

3. Albeverio, S., Høegh-Krohn, R.: The Wightman axioms and the mass gap for strong interactions of exponential type in two-dimensional space time. Preprint. Math, Inst. University of Oslo, No 12, 1973, J. Funct. Anal. 16, 39-82 (1974) 
4. Nelson, E.: J. Funct. Anal. 12, 211-227 (1973)

5. Guerra,F., Rosen,L., Simon,B.: The $P(\varphi)_{2}$ Euclidean quantum field theory as a classical statistical mechanics. Preprint 1973, Princeton University

6. Dobrushin,R.L., Minlos, R.A.: Construction of a one dimensional quantum field via a continuous Markov field. Submitted to: Functional analysis and its applications, Moscow

7. Glimm,J., Jaffe, A.: Phys. Rev. 176, 1945-1951 (1968)

8. Glimm, J., Jaffe, A.: Ann. Math. 91, 362-401 (1970)

9. Glimm, J., Jaffe, A.: Acta Math. 125, 203-261 (1970)

10. Rosen, L.: Commun. math. Phys. 16, 157-183 (1970)

11. Høegh-Krohn, R.: Commun. math. Phys. 21, 244-255 (1972)

12. Nelson, E.: Probability theory and Euclidean field theory. In: Velo, G., Wightman, A. (Eds.): Constructive quantum field theory. Berlin-Heidelberg-New York: Springer 1973

13. Simon, B., Høegh-Krohn, R.: J. Funct. Anal. 9, 121-180 (1972)

14. a) Guerra,F.: Phys. Rev. Letters 28, 1213-1215 (1972)

b) Guerra, F., Rosen, L., Simon, B.: Commun. math. Phys. 27, 10-22 (1972)

c) Guerra, F., Rosen, L., Simon, B.: Commun. math. Phys. 29, 233-247 (1973)

Communicated by R. Haag and A. S. Wightman Raphael Høegh-Krohn

Universitetet i Oslo

Det Matematik-

Natureibenskaplige

Postboks 1032

Blindern, Oslo 3, Norway 